\title{
EXISTENCE DE NAPPES DE TOURBILLON EN DIMENSION DEUX
}

\author{
JEAN-MARC DELORT
}

\section{INTRODUCTION}

Cet article est consacré à la preuve d'un résultat d'existence de nappes de tourbillon en dimension 2. Le problème des nappes de tourbillon consiste à trouver une solution $(v, p)$, définie sur $\mathbb{R} \times \mathbb{R}^{2}$, muni des coordonnées $(t, x=$ $\left.\left(x_{1}, x_{2}\right)\right)$, à valeurs dans $\mathbb{R}^{2} \times \mathbb{R}$, à l'équation d'Euler

$$
\left\{\begin{array}{l}
\frac{\partial v}{\partial t}+\operatorname{div}(v \otimes v)=-\nabla p, \\
\operatorname{div} v=0,
\end{array}\right.
$$

(cf $\S 1.1$ pour les notations utilisées ci-dessus), dont la donnée initiale $\left.v\right|_{t=0}=v_{0}$ vérifie $\operatorname{div} v_{0}=0$ et a un tourbillon $\omega_{0}=\operatorname{rot} v_{0}$ de la forme $\omega_{0}=\delta_{\Sigma}$, avec $\Sigma$ courbe compacte lisse de $\mathbb{R}^{2}$. Plus généralement, on cherche des solutions dont le tourbillon initial $\omega_{0}$ est une mesure à support compact, appartenant à l'espace de Sobolev $H^{-1}\left(\mathbb{R}^{2}\right)$ (ce qui entraîne que $v_{0}$ est localement dans $L^{2}$ ).

Ce problème a été abordé par plusieurs auteurs. La méthode classique d'attaque consiste à régulariser la donnée initiale, et à utiliser que, pour chaque régularisée, le problème $(*)$ a une solution globale. On obtient ainsi une suite de solutions $C^{\infty}\left(v^{\varepsilon}, p^{\varepsilon}\right)$ et $v^{\varepsilon}$ admet des estimations uniformes en $\varepsilon$ (cf. [4]). Cela permet d'extraire une sous-suite, convergeant faiblement dans $L_{\text {loc }}^{2}$ vers une limite $v$, et le problème consiste à prouver que l'on peut passer à la limite dans l'équation, i.e., qu'il existe $p$ tel que $(v, p)$ vérifie $(*)$. La difficulté provient évidemment du fait que la convergence n'étant en général pas forte, $v^{\varepsilon} \otimes v^{\varepsilon}$ converge au sens des distributions non pas vers $v \otimes v$ mais vers $v \otimes v+\nu$, où $\nu$ est une matrice $2 \times 2$ à coefficients mesures.

Donnons maintenant quelques indications sur la genèse historique du sujet. Dans [5] Di Perna-Majda ont introduit la notion de "mesure de défaut réduite" d'une suite de $L_{\text {loc }}^{\infty}\left(\mathbb{R}, L_{\text {loc }}^{2}\left(\mathbb{R}^{2} ; \mathbb{R}^{2}\right)\right)$. Ils ont prouvé qu'une suite de solutions de $(*)$, bornée dans l'espace précédent, et faiblement convergente, a sa mesure de défaut réduite concentrée dans un ensemble petit (au sens de la dimension de Hausdorff) [5, Théorème 1]. En outre, [5, Théorème 2], une hypothèse de

Received by the editors November 2, 1990 and, in revised form, March 30, 1991.

1991 Mathematics Subject Classification. Primary 35L60, 76C05. 
petitesse plus forte sur cet ensemble de concentration entraîne que la limite faible est solution de $(*)$ (bien que la convergence ne soit pas nécessairement forte). Cette dernière hypothèse peut être affaiblie dans le cas de suites de solutions stationnaires [5, Théorème 3.1]. Cette condition faible est, pour de telles suites, automatiquement vérifiée dès que l'on rajoute à l'hypothèse de borne uniforme de la vitesse dans $L_{\text {loc }}^{2}$ une condition de majoration uniforme du tourbillon dans $L^{1}$ [5, Théorème 3], analogue à celle dont nous disposerons ci-dessous.

D'autre part, Krasny $[13,14]$ a étudié numériquement le problème $(*)$ avec une donnée de la forme $\varphi \delta_{\Sigma}$ où $\varphi$ est une fonction réelle. Ses résultats indiquent que le comportement de l'approximation numérique du tourbillon de la solution à un instant donné est beaucoup plus compliqué lorsque $\varphi$ peut changer de signe que lorsque $\varphi$ conserve un signe fixe. En analysant cette étude, couplée à certains résultats découlant du Théorème 3.1 de [4], Majda [15, 16] conjecture que le problème $(*)$ admet une solution globale lorsqu'on rajoute l'hypothèse supplémentaire que $\omega_{0}$ est de signe fixe-hypothèse évidemment satisfaite par des données de la forme $\omega_{0}=\delta_{\Sigma}$. Nous nous proposons ici de prouver ce résultat. La méthode consiste d'abord à remarquer qu'il suffit d'étudier non pas la limite de $v^{\varepsilon} \otimes v^{\varepsilon}$ mais seulement celle de certaines combinaisons linéaires des coefficients de cette matrice. Plus précisément, en exprimant la pression $p^{\varepsilon}$ à l'aide de la vitesse $v^{\varepsilon}$ par la relation $-\Delta p^{\varepsilon}=\operatorname{div} \operatorname{div}\left(v^{\varepsilon} \otimes v^{\varepsilon}\right.$ ) (obtenue en prenant la divergence de la première équation $(*)$ compte tenu de la seconde) et en reportant la valeur trouvée dans $(*)$, on montre que $\left(v^{\varepsilon}, p^{\varepsilon}\right)$ vérifie $(*)$ si et seulement si $v^{\varepsilon}={ }^{t}\left(v_{1}^{\varepsilon}, v_{2}^{\varepsilon}\right)$ satisfait

$$
\frac{\partial v^{\varepsilon}}{\partial t}+\Delta^{-1} A(D)\left(\begin{array}{c}
\left(v_{1}^{\varepsilon}\right)^{2}-\left(v_{2}^{\varepsilon}\right)^{2} \\
v_{1}^{\varepsilon} \cdot v_{2}^{\varepsilon}
\end{array}\right)=0
$$

où $A(D)$ est un opérateur différentiel matriciel homogène de degré 3 (cf. Lemme 1.1.2).

Pour prouver que $v$ vérifie $(*)$ pour un certain $p$, il suffit donc de voir que $v$ satisfait $(* *)$ et donc il suffit de montrer que $\lim v_{1}^{\varepsilon} \cdot v_{2}^{\varepsilon}=v_{1} \cdot v_{2}$ et $\lim \left(\left(v_{1}^{\varepsilon}\right)^{2}-\left(v_{2}^{\varepsilon}\right)^{2}\right)=v_{1}^{2}-v_{2}^{2}$.

Ces égalités sont prouvées au paragraphe 1.2, en exprimant, pour toute fonction test $\varphi \in C_{0}^{\infty}\left(\mathbb{R}^{2}\right)$, l'intégrale $\int v_{1}^{\varepsilon}(t, z) v_{2}^{\varepsilon}(t, z) \varphi(z) d z$ à l'aide du tourbillon $\omega^{\varepsilon}=\operatorname{rot} v^{\varepsilon}$, par la relation $v^{\varepsilon}=\nabla^{\perp} \Delta^{-1} \omega^{\varepsilon}$. L'intégrale étudiée s'écrit alors

$$
\int \omega^{\varepsilon}(t, x) \omega^{\varepsilon}(t, y) H_{\varphi}(x, y) d x d y
$$

où $H_{\varphi}$ est une distribution s'écrivant explicitement à l'aide de $\varphi$ et de la solution élémentaire du laplacien. Le point clef-qui est celui faisant intervenir le fait que l'on soit parti d'une non linéarité $v_{1}^{\varepsilon} \cdot v_{2}^{\varepsilon}\left(\right.$ ou $\left.\left(v_{1}^{\varepsilon}\right)^{2}-\left(v_{2}^{\varepsilon}\right)^{2}\right)$ et non pas, par exemple, de $\left(v_{1}^{\varepsilon}\right)^{2}$-consiste à prouver que $H_{\varphi}$ est une fonction bornée. Cette dernière propriété, combinée au fait qu'une mesure dans $H^{-1}\left(\mathbb{R}^{2}\right)$ est nécessairement diffuse et à la positivité (ou la négativité) de $\omega_{0}$ permet alors 
de passer à la limite dans l'expression $(* * *)$. L'argument essentiel est le Lemme 1.2.7 ci-dessous, qui est le seul endroit de la preuve où est utilisée l'hypothèse de signe fixe sur $\omega_{0}$.

En résumé, le cœur de la méthode consiste donc à montrer que, sous les hypothèses faites, la limite faible, à extraction éventuelle près d'une sous-suite, de $v^{\varepsilon} \otimes v^{\varepsilon}-v \otimes v$ est une matrice scalaire.

A cet égard, remarquons que le fait qu'il suffise d'étudier un certain type de non-linéarités quadratiques, i.e. le fait que $(*)$ puisse se mettre sous la forme $(* *)$, apparaît chez Majda [5, pp. 71 et suivantes] et dans d'autres travaux ultérieurs $[2,12]$.

D'autre part, signalons que la méthode consistant à exprimer dans l'équation d'Euler la pression en fonction de la vitesse a également été utilisée par Chemin [3] pour l'étude de solutions ayant une plus grande régularité que celles étudiées ici (la pression peut alors être exprimée à partir d'un opérateur pseudodifférentiel non-linéaire d'ordre -1 agissant sur la vitesse).

Un certain nombre d'exemples explicites de suites $\left(v^{\varepsilon}\right)_{\varepsilon \in] 0,1]}$ de solutions stationnaires vérifiant les hypothèses de borne uniforme indiquées ci-dessus convergeant faiblement (mais pas fortement) vers 0 ont été construits dans [4, 8]. Dans tous ces exemples, les non-linéarités quadratiques $v_{1}^{\varepsilon} \cdot v_{2}^{\varepsilon}$ et $\left(v_{1}^{\varepsilon}\right)^{2}-\left(v_{2}^{\varepsilon}\right)^{2}$ passent à la limite lorsque $\varepsilon \rightarrow 0$, que l'hypothèse de positivité sur le tourbillon que nous faisons ici soit satisfaite ou non.

D'après [4] le problème $(*)$ avec une donnée à tourbillon $\omega_{0} \in L_{\text {comp }}^{p}\left(\mathbb{R}^{2}\right)$ $(p \in] 1,+\infty])$ admet une solution globale. En combinant les arguments permettant de prouver ce résultat avec la méthode que nous avons indiquée ci-dessus, il est possible d'étendre le résultat d'existence que nous venons de décrire à une donnée dont le tourbillon s'écrit $\omega_{0}=\omega_{0}^{\prime}+\omega_{0}^{\prime \prime}$, où $\omega_{0}^{\prime}$ est une mesure de signe fixe dans $H_{\text {comp }}^{-1}\left(\mathbb{R}^{2}\right)$, et où $\omega_{0}^{\prime \prime} \in L_{\text {comp }}^{p}\left(\mathbb{R}^{2}\right)$ avec $p>1$. Dans la deuxième section de ce travail, nous prouvons que l'analogue de ce théorème sur une surface riemannienne compacte, connexe, orientée, sans bord $(M, g)$, est également vrai (sous réserve que soit de plus vérifiée la condition nécessaire évidente $\int_{M} \omega_{0}=0$.) La méthode est identique à celle utilisée sur le plan. On doit toutefois obtenir des expressions intrinsèques pour les diverses quantités manipulées. En particulier, les non-linéarités quadratiques sur lesquelles on doit passer à la limite sont les coefficients du tenseur de type $(1,1)$

$$
v^{\varepsilon} \otimes \tilde{v}^{\varepsilon}-\frac{1}{2} g\left(v^{\varepsilon}, v^{\varepsilon}\right) \delta
$$

où $v^{\varepsilon}$ est la suite de champs de vecteurs solutions de l'équation à données régularisées, $\tilde{v}^{\varepsilon}$ la suite de 1 -formes lui correspondant par l'identification de $T M$ à $T^{*} M$ déduite de la métrique, et $\delta$ le tenseur donné en coordonnées locales par

$$
\delta=\delta_{j}^{i} \frac{\partial}{\partial x^{i}} \otimes d x^{j} \quad\left(\delta_{j}^{i} \text { symbole de Kronecker }\right) .
$$

Je remercie S. Alinhac, P. Gérard, G. Lebeau, et G. Métivier pour des discussions sur ce travail. Je remercie également le referee pour de minutieux 
commentaires historiques et pour m'avoir signalé les références $[15,16]$ de la bibliographie.

\section{NAPPES DE TOURBILLON SUR $\mathbb{R}^{2}$}

1.1. Enoncé du théorème. On note $x=\left(x_{1}, x_{2}\right)$ les coordonnées sur $\mathbb{R}^{2}$. Si $\varphi$ (resp. $v={ }^{t}\left(v_{1}, v_{2}\right)$, resp. $\left.M=\left(m_{i j}\right)_{1 \leq i, j \leq 2}\right)$ est une distribution sur $\mathbb{R}^{2}$ à valeurs dans $\mathbb{R}$ (resp. dans $\mathbb{R}^{2}$, resp. dans l'anneau des matrices carrées d'ordre 2) on notera:

$$
\begin{aligned}
\partial_{j} \varphi & =\frac{\partial \varphi}{\partial x_{j}}, \quad D_{j} \varphi=\frac{1}{i} \frac{\partial \varphi}{\partial x_{j}}, \quad j=1,2 ; \\
\nabla \varphi & ={ }^{t}\left(\partial_{1} \varphi, \partial_{2} \varphi\right), \quad \nabla^{\perp} \varphi={ }^{t}\left(-\partial_{2} \varphi, \partial_{1} \varphi\right), \\
\Delta \varphi & =\left(\partial_{1}^{2}+\partial_{2}^{2}\right) \varphi
\end{aligned}
$$

(resp.

$$
\operatorname{div} v=\partial_{1} v_{1}+\partial_{2} v_{2}, \quad \operatorname{rot} v=\partial_{1} v_{2}-\partial_{2} v_{1}
$$

resp.

$$
\left.\operatorname{div} M={ }^{t}\left(\partial_{1} m_{11}+\partial_{2} m_{12}, \partial_{1} m_{21}+\partial_{2} m_{22}\right)\right) \text {. }
$$

Si $\varphi$ est une distribution tempérée sur $\mathbb{R}^{2}$, à valeurs réelles, telle que sa transformée de Fourier $\hat{\varphi}(\xi)$ soit $L_{\text {loc }}^{\infty}$ (resp. $L_{\text {loc }}^{1}$ ) au voisinage de 0 et si $P(D)$ est un opérateur différentiel homogène à coefficients constants, d'ordre 1 (resp. 2) on pose

$$
P(D) \Delta^{-1} \varphi=\Delta^{-1} P(D) \varphi \stackrel{\text { def }}{=} \mathscr{F}^{-1}\left(-\frac{P(\xi)}{|\xi|^{2}} \hat{\varphi}(\xi)\right),
$$

où $\mathscr{F}^{-1}$ est la transformation de Fourier inverse et où le produit à l'intérieur de son argument a un sens.

Si $\varphi$ est une distribution à support compact sur $\mathbb{R}^{2}$, on posera également

$$
\Delta^{-1} \varphi=k * \varphi,
$$

où $k(x)=\frac{1}{2 \pi} \log |x|$ est la solution élémentaire du laplacien. Bien entendu les relations (1.1.4) et (1.1.5) sont compatibles.

On notera $\mathscr{D}^{\prime}\left(\mathbb{R}^{2}\right), L^{2}\left(\mathbb{R}^{2}\right), \ldots$ les espaces de distributions à valeurs scalaires et $\mathscr{D}^{\prime}\left(\mathbb{R}^{2} ; \mathbb{R}^{2}\right), L^{2}\left(\mathbb{R}^{2} ; \mathbb{R}^{2}\right), \ldots$ les espaces de distributions à valeurs dans $\mathbb{R}^{2}$. Enfin si $v \in L_{\text {loc }}^{2}\left(\mathbb{R}^{2} ; \mathbb{R}^{2}\right)$, on notera $v \otimes v$ la matrices $\left(v_{i} v_{j}\right)_{1 \leq i, j \leq 2}$.

Théorème 1.1.1. Soient $\omega_{0}$ une mesure de Radon positive à support compact sur $\mathbb{R}^{2}$ qui est dans l'espace de Sobolev $H^{-1}\left(\mathbb{R}^{2}\right)$ et $v_{0}=\nabla^{\perp} \Delta^{-1} \omega_{0}$. Il existe une fonction $v \in L_{\text {loc }}^{\infty}\left(\mathbb{R} ; L_{\text {loc }}^{2}\left(\mathbb{R}^{2} ; \mathbb{R}^{2}\right)\right)$ et une fonction $p \in L_{\text {loc }}^{\infty}\left(\mathbb{R} ; \mathscr{S}^{\prime}\left(\mathbb{R}^{2}\right)\right)$ solutions $d u$ système d'Euler

$$
\left\{\begin{array}{l}
\partial v / \partial t+\operatorname{div}(v \otimes v)=-\nabla p \\
\operatorname{div} v=0 \\
\left.v\right|_{t=0}=v_{0}
\end{array}\right.
$$


De plus, $v(t, x)$ s'écrit $v(t, x)=\bar{v}(x)+\check{v}(t, x)$ où $\bar{v}=\nabla^{\perp} \Delta^{-1} \bar{\omega}$ avec $\bar{\omega} \epsilon$ $C_{0}^{\infty}\left(\mathbb{R}^{2}\right)$ radiale vérifiant $\int \bar{\omega}(x) d x=\int \omega_{0}$ et où $\check{v} \in L_{\mathrm{loc}}^{\infty}\left(\mathbb{R} ; L^{2}\left(\mathbb{R}^{2} ; \mathbb{R}^{2}\right)\right)$.

En outre $\omega(t, \cdot)=\operatorname{rot} v(t, \cdot)$ est pour tout $t \in \mathbb{R}$ une mesure positive de masse totale uniformément bornée en $t$ par $\int \omega_{0}$.

Enfin la transformée de Fourier de $p$ en $x, \hat{p}(t, \xi)$ peut être supposé dans $L_{\text {loc }}^{\infty}\left(\mathbb{R} ; L_{\text {loc }}^{2}\left(\mathbb{R}^{2}\right)\right)$.

Remarque. Dans l'énoncé ci-dessus, le fait que $\bar{\omega}$ soit une fonction radiale entraîne que $\bar{v}$ est solution de l'équation d'Euler stationnaire. D'autre part, la condition de régularité sur $\hat{p}(t, \xi)$ n'est qu'une manière d'exprimer une certaine décroissance de $p(t, x)$ lorsque $x \rightarrow \infty$. Enfin, l'équation étant non caractéristique pour les hyperplans $t=$ cst, $v \in C^{0}\left(\mathbb{R} ; \mathscr{D}^{\prime}\left(\mathbb{R}^{2} ; \mathbb{R}^{2}\right)\right)$ ce qui donne un sens à la condition initiale (qui vérifie par construction la contrainte $\left.\operatorname{div} v_{0}=0\right)$.

Remarque. Le même énoncé est valable en remplaçant la condition $\omega_{0} \geq 0$ par $\omega_{0} \leq 0$ : il suffit pour le voir de remplacer dans (1.1.6) $v$ par $-v$ et $t$ par $-t$.

On peut légèrement généraliser le Théorème 1.1 .1 en rajoutant à la donnée mesure positive une perturbation plus régulière mais ne vérifiant aucune condition de signe. D'après Di Perna-Majda [4], on sait que le problème (1.1.6) avec une donnée $v_{0}$ ayant un rotationnel $L^{p}$ à support compact avec $p>1$ admet une solution globale. On peut donner un énoncé combinant ce résultat avec le Théorème 1.1.1.

Théorème 1.1.1'. Soient $\omega_{0}^{\prime}$ une mesure de Radon positive à support compact sur $\mathbb{R}^{2}$ qui est dans l'espace de Sobolev $H^{-1}\left(\mathbb{R}^{2}\right)$ et $\omega_{0}^{\prime \prime}$ un élément de $L^{p}\left(\mathbb{R}^{2}\right)$ à support compact, avec $p>1$. Posons $\omega_{0}=\omega_{0}^{\prime}+\omega_{0}^{\prime \prime}, v_{0}=\nabla^{\perp} \Delta^{-1} \omega_{0}$. Il existe une fonction $v \in L_{\text {loc }}^{\infty}\left(\mathbb{R} ; L_{\text {loc }}^{2}\left(\mathbb{R}^{2} ; \mathbb{R}^{2}\right)\right)$ et une fonction $p \in L_{\text {loc }}^{\infty}\left(\mathbb{R} ; \mathscr{S}^{\prime}\left(\mathbb{R}^{2}\right)\right)$ solutions de (1.1.6). De plus, $v(t, x)$ s'écrit $v(t, x)=\bar{v}(x)+\check{v}(t, x)$ où $\bar{v}=\nabla^{\perp} \Delta^{-1} \bar{\omega}$ avec $\bar{\omega} \in C_{0}^{\infty}\left(\mathbb{R}^{2}\right)$ radiale vérifiant $\int \bar{\omega}=\int \omega_{0}$ et où $\check{v} \in$ $L_{\text {loc }}^{\infty}\left(\mathbb{R} ; L^{2}\left(\mathbb{R}^{2} ; \mathbb{R}^{2}\right)\right)$.

En outre $\omega(t, x)=\operatorname{rot} v(t, x)$ s'écrit $\omega=\omega^{\prime}+\omega^{\prime \prime}$ avec $\omega^{\prime}$ fonction $L^{\infty}$ de $t$ à valeurs dans les mesures positives ou nulles, de masse totale uniformément bornée en $t$ par $\int \omega_{0}^{\prime}$ et $\omega^{\prime \prime} \in L^{\infty}\left(\mathbb{R}, L^{q}\left(\mathbb{R}^{2}\right)\right)$ pour tout $q \in[1, p]$.

Enfin $\hat{p}(t, \xi)$ peut être supposé dans $L_{\text {loc }}^{\infty}\left(\mathbb{R}, L_{\text {loc }}^{2}\left(\mathbb{R}^{2}\right)\right)$.

La preuve du théorème repose sur l'écriture du système équivalent à (1.1.6) obtenu en exprimant la pression $p$ à partir de la vitesse $v$ (cf. également [5, $\S 3$, p. 71]).

Lemme 1.1.2. Soient $\bar{v}$ une fonction continue sur $\mathbb{R}^{2}$ à valeurs dans $\mathbb{R}^{2}$ telle que $(1+|x|) \bar{v}$ soit bornée sur $\mathbb{R}^{2}$ et $(t, x) \rightarrow \check{v}(t, x)$ un élément de $L_{\text {loc }}^{\infty}\left(\mathbb{R}, L^{2}\left(\mathbb{R}^{2} ; \mathbb{R}^{2}\right)\right)$. Soit $v(t, x)=\bar{v}(x)+\check{v}(t, x)$. Les conditions suivantes 
sont équivalentes:

(i) Il existe une distribution $p \in L_{\mathrm{loc}}^{\infty}\left(\mathbb{R}, \mathscr{S}^{\prime}\left(\mathbb{R}^{2}\right)\right)$ telle que $\hat{p}$ soit dans $L_{\mathrm{loc}}^{\infty}\left(\mathbb{R}, L_{\mathrm{loc}}^{2}\left(\mathbb{R}^{2}\right)\right)$ et que $(v, p)$ soit solution de

$$
\left\{\begin{array}{l}
\partial v / \partial t+\operatorname{div}(v \otimes v)=-\nabla p \\
\operatorname{div} v=0
\end{array}\right.
$$

(ii) La distribution $v$ vérifie

$$
\left\{\begin{array}{l}
\partial v / \partial t+\Delta^{-1} A(D)\left(\begin{array}{c}
v_{1}^{2}-v_{2}^{2} \\
v_{1} v_{2}
\end{array}\right)=0, \\
\left.\operatorname{div} v\right|_{t=0}=0,
\end{array}\right.
$$

où $A(D)$ est l'opérateur différentiel matriciel

$$
A(D)=\left(\begin{array}{cc}
\partial_{1} \partial_{2}^{2} & \partial_{2}\left(\partial_{2}^{2}-\partial_{1}^{2}\right) \\
-\partial_{1}^{2} \partial_{2} & \partial_{1}\left(\partial_{1}^{2}-\partial_{2}^{2}\right)
\end{array}\right) .
$$

Démonstration. Supposons (i) et prouvons (ii). Par hypothèse, pour tous $1 \leq$ $i, j \leq 2, v_{i} v_{j} \in L_{\text {loc }}^{\infty}\left(\mathbb{R}, L^{1}\left(\mathbb{R}^{2}\right)+L^{2}\left(\mathbb{R}^{2}\right)\right)$. Prenant la divergence de la première équation (1.1.7) et utilisant la seconde, on obtient $-\Delta p=\operatorname{div} \operatorname{div}(v \otimes v)$ d'où

$$
p=-\Delta^{-1} \operatorname{div} \operatorname{div}(v \otimes v) .
$$

Reportant cette valeur dans (1.1.7), on obtient

$$
\partial v / \partial t+\left(\operatorname{Id}-\nabla \Delta^{-1} \operatorname{div}\right) \operatorname{div}(v \otimes v)=0 .
$$

Or (Id $-\nabla \Delta^{-1}$ div) n'est autre que l'opérateur différentiel matriciel (agissant sur les champs de vecteurs)

$$
\Delta^{-1}\left(\begin{array}{cc}
\partial_{2}^{2} & -\partial_{1} \partial_{2} \\
-\partial_{1} \partial_{2} & \partial_{1}^{2}
\end{array}\right)
$$

Si l'on fait agir cet opérateur sur $\operatorname{div}(v \otimes v)$ un calcul immédiat montre que l'on obtient $\Delta^{-1} A(D) \cdot{ }^{t}\left(v_{1}^{2}-v_{2}^{2}, v_{1} v_{2}\right)$. L'assertion (ii) est donc vérifiée.

Réciproquement, si (ii) est vérifié, définissons une fonction $p$ par (1.1.10). Elle vérifie les conditions de régularité énoncées dans (i) et d'après (1.1.11), (1.1.12) la première équation (1.1.7) est satisfaite. D'autre part, il résulte de la première équation (1.1.8) et de la forme de $A(D)$ que $(\partial / \partial t) \operatorname{div} v=0$. La seconde équation (1.1.8) entraîne donc la seconde équation (1.1.7). Le lemme est donc prouvé.

Nous allons maintenant montrer que le lemme précédent réduit la preuve du théorème à l'obtention d'un résultat de passage à la limite faible sur des expressions de la forme $\left(v_{1}^{\varepsilon}\right)^{2}-\left(v_{2}^{\varepsilon}\right)^{2}$ et $v_{1}^{\varepsilon} \cdot v_{2}^{\varepsilon}$.

Pour cela, choisissons une fonction $\bar{\omega}(x), C^{\infty}$ à support compact sur $\mathbb{R}^{2}$, radiale, telle que $\int \bar{\omega}(x) d x=\int \omega_{0}$ et posons $\bar{v}=\nabla^{\perp} \Delta^{-1} \bar{\omega}$. Alors $\bar{v}$ est $C^{\infty}$ sur $\mathbb{R}^{2}$ et est $O(1 /|x|)$ lorsque $x \rightarrow \infty$. On notera $\check{\omega}_{0}=\omega_{0}-\bar{\omega}$, on a $\int \check{\omega}_{0}=$ 0 . Fixons $\rho \in C_{0}^{\infty}\left(\mathbb{R}^{2}\right)$ radiale, positive ou nulle, vérifiant $\int \rho(x) d x=1$ et 
posons pour $\varepsilon \in] 0,1], \rho_{\varepsilon}(x)=\varepsilon^{-2} \rho(x / \varepsilon)$ et

$$
\omega_{0}^{\varepsilon}=\omega_{0} * \rho_{\varepsilon}, \quad \check{\omega}_{0}^{\varepsilon}=\omega_{0}^{\varepsilon}-\bar{\omega}, \quad \omega_{0}^{\varepsilon}=\omega_{0}^{\prime} * \rho_{\varepsilon}, \quad \omega_{0}^{\prime \prime \varepsilon}=\omega_{0}^{\prime \prime} * \rho_{\varepsilon} .
$$

Alors $\left(\omega_{0}^{\varepsilon}\right)_{\varepsilon \in] 0,1]},\left(\check{\omega}_{0}^{\varepsilon}\right)_{\varepsilon \in] 0,1]},\left(\omega_{0}^{\prime \varepsilon}\right)_{\varepsilon \in] 0,1]},\left(\omega_{0}^{\prime \prime}\right)_{\varepsilon \in] 0,1]}$ sont des familles de fonctions $C^{\infty}$ à support contenu dans un compact uniforme de $\mathbb{R}^{2}$ et on a

$$
\begin{aligned}
& \int\left|\omega_{0}^{\varepsilon}\right| d x \leq \int\left|\omega_{0}\right|, \quad \int\left|\check{\omega}_{0}^{\varepsilon}\right| d x \leq \int\left|\omega_{0}\right|+\int|\bar{\omega}(x)| d x, \\
& \int \omega_{0}^{\prime \varepsilon} d x=\int \omega_{0}^{\prime}, \quad\left\|\omega_{0}^{\prime \prime}\right\|_{L^{p}} \leq\left\|\omega_{0}^{\prime \prime}\right\|_{L^{p}} .
\end{aligned}
$$

En outre, $\omega_{0}^{\varepsilon} \geq 0$ et lorsque $\varepsilon$ tend vers $0, \omega_{0}^{\varepsilon}$ (resp. $\check{\omega}_{0}^{\varepsilon}$, resp. $\omega_{0}^{\prime \varepsilon}$, resp. $\left.\omega_{0}^{\prime \prime}\right)$ converge fortement dans $H^{-1}\left(\mathbb{R}^{2}\right)$ vers $\omega_{0}$ (resp. $\check{\omega}_{0}$, resp. $\omega_{0}^{\prime}$, resp. $\omega_{0}^{\prime \prime}$ (en utilisant que $L_{\text {comp }}^{p}\left(\mathbb{R}^{2}\right) \hookrightarrow H^{-1}\left(\mathbb{R}^{2}\right)$ pour $\left.p>1\right)$ ).

On pose

$$
\begin{gathered}
v_{0}^{\prime \varepsilon}=\nabla^{\perp} \Delta^{-1} \omega_{0}^{\varepsilon}, \quad v_{0}^{\prime \prime \varepsilon}=\nabla^{\perp} \Delta^{-1} \omega_{0}^{\prime \prime \varepsilon}, \\
\check{v}_{0}^{\varepsilon}=\nabla^{\perp} \Delta^{-1} \check{\omega}_{0}^{\varepsilon}, \quad v_{0}^{\varepsilon}=\bar{v}+\check{v}_{0}^{\varepsilon}=\nabla^{\perp} \Delta^{-1} \omega_{0}^{\varepsilon} .
\end{gathered}
$$

Comme la transformée de Fourier de $\check{\omega}_{0}^{\varepsilon}$ est nulle en 0 et que $\check{\omega}_{0}^{\varepsilon}$ est uniformément bornée dans $H^{-1}\left(\mathbb{R}^{2}\right) \cap L^{1}\left(\mathbb{R}^{2}\right)$ à support dans un compact fixe, on voit immédiatement que $\left(\check{v}_{0}^{\varepsilon}\right)_{\varepsilon \in] 0,1]}$ est une famille de fonctions $C^{\infty}$ sur $\mathbb{R}^{2}$, uniformément bornée dans $L^{2}\left(\mathbb{R}^{2} ; \mathbb{R}^{2}\right)$.

Pour tout $\varepsilon \in] 0,1]$ la fonction $v_{0}^{\varepsilon}$ est donc $C^{\infty}$ et son rotationnel $\omega_{0}^{\varepsilon}$ est $C^{\infty}$ à support compact. Il est alors bien connu (cf. [10; $4 ; 3$, Théorème $\left.\mathrm{A}\right]$ ) que le problème suivant, équivalent à (1.1.6) pour les solutions régulières, et dans lequel on désigne par $v \cdot \nabla$ l'opérateur $v_{1} \partial_{1}+v_{2} \partial_{2}$

$$
\left\{\begin{array}{l}
\partial v^{\varepsilon} / \partial t+\left(v^{\varepsilon} \cdot \nabla\right) v^{\varepsilon}=-\nabla p^{\varepsilon} \\
\operatorname{div} v^{\varepsilon}=0 \\
\left.v^{\varepsilon}\right|_{t=0}=v_{0}^{\varepsilon}
\end{array}\right.
$$

admet pour tout $\varepsilon \in] 0,1]$ une solution globale $C^{\infty}, v^{\varepsilon}(t, x)=\bar{v}(x)+\check{v}^{\varepsilon}(t, x)$ où $\check{v}^{\varepsilon} \in C^{0}\left(\mathbb{R} ; H^{s}\left(\mathbb{R}^{2} ; \mathbb{R}^{2}\right)\right)$ pour tout $s \in \mathbb{R}$. Il est classique que pour tout $T>0$, il existe $C_{T}>0$ tel que

$$
\sup _{\varepsilon \in] 0,1]} \sup _{t \in[-T, T]}\left\|\check{v}^{\varepsilon}(t, \cdot)\right\|_{L^{2}\left(\mathbb{R}^{2} ; \mathbb{R}^{2}\right)} \leq C_{T} .
$$

Il suffit en effet de remarquer que

$$
\frac{d}{d t}\left\|\check{v}^{\varepsilon}(t, \cdot)\right\|_{L^{2}\left(\mathbb{R}^{2} ; \mathbb{R}^{2}\right)}^{2}=2\left(\frac{d \check{v}^{\varepsilon}}{d t}, \check{v}^{\varepsilon}\right)=-2\left(\left(\check{v}^{\varepsilon} \cdot \nabla\right) \bar{v}, \check{v}^{\varepsilon}\right)
$$

en utilisant la première équation (1.1.16) et le fait que si $w$ est un champ de vecteurs à divergence nulle, à coefficients réels $C^{\infty}$ bornés et si $\theta \in H^{1}\left(\mathbb{R}^{2}\right)$ on a $((w \cdot \nabla) \theta, \theta)=0$. L'inégalité (1.1.17) résulte alors du lemme de Gronwall. 
D'autre part, posons

$$
\omega^{\varepsilon}(t, x)=\operatorname{rot}_{x} v^{\varepsilon}(t, x) .
$$

D'après ce qui précède, $\omega^{\varepsilon}$ est donc une famille de fonctions de $L_{\text {loc }}^{\infty}\left(\mathbb{R}, C^{\infty}\left(\mathbb{R}^{2}\right)\right)$ bornée dans $L_{\text {loc }}^{\infty}\left(\mathbb{R}, H^{-1}\left(\mathbb{R}^{2}\right)\right)$. En outre, il découle classiquement de l'équation (1.1.16) que $\omega^{\varepsilon}$ vérifie

$$
\left\{\begin{array}{l}
\partial \omega^{\varepsilon} / \partial t+\left(v^{\varepsilon} \cdot \nabla\right) \omega^{\varepsilon}=0, \\
\left.\omega^{\varepsilon}\right|_{t=0}=\omega_{0}^{\varepsilon}
\end{array}\right.
$$

La fonction $\omega^{\varepsilon}$ est donc constante le long des courbes intégrales du champ de vecteurs $\partial / \partial t+v^{\varepsilon} \cdot \nabla$. Soit $\phi^{\varepsilon}$ la solution de

$$
\left\{\begin{array}{l}
\partial \phi^{\varepsilon} / \partial t=v^{\varepsilon}\left(t, \phi^{\varepsilon}(t, x)\right), \\
\phi^{\varepsilon}(0, x)=x .
\end{array}\right.
$$

D'après $(1.1 .20)$ on a $\omega_{0}^{\varepsilon}(x)=\omega^{\varepsilon}\left(t, \phi^{\varepsilon}(t, x)\right)$ et en décomposant $\omega_{0}^{\varepsilon}$ en $\omega_{0}^{\varepsilon}+$ $\omega_{0}^{\prime \prime \varepsilon}$ on écrit $\omega^{\varepsilon}(t, x)=\omega^{\varepsilon}(t, x)+\omega^{\prime \prime \varepsilon}(t, x)$ avec

$$
\omega_{0}^{\prime \varepsilon}(x)=\omega^{\prime \varepsilon}\left(t, \phi^{\varepsilon}(t, x)\right), \quad \omega_{0}^{\prime \prime \varepsilon}(x)=\omega^{\prime \prime \varepsilon}\left(t, \phi^{\varepsilon}(t, x)\right) .
$$

Pour tout $t \in \mathbb{R}$ et tout $\varepsilon \in] 0,1]$ fixés, $\omega^{\varepsilon}, \omega^{\prime}, \omega^{\prime \prime}$ sont des fonctions $C^{\infty}$ à support compact en $x$ et $\omega^{\prime \varepsilon}$ est positive ou nulle. D'autre part, pour tout $t \in \mathbb{R}$ le jacobien de $x \rightarrow \phi^{\varepsilon}(t, x)$ est identiquement égal à 1 (comme on le voit en utilisant $(1.1 .21)$ et la relation $\left.\operatorname{div} v^{\varepsilon}=0\right)$. Il résulte alors de (1.1.14) que pour tout $t \in \mathbb{R}$

$$
\begin{aligned}
\int \omega^{\prime \varepsilon}(t, x) d x & =\int \omega_{0}^{\prime \varepsilon}(x) d x=\int \omega_{0}^{\prime}, \\
\left(\int\left|\omega^{\prime \prime \varepsilon}(t, x)\right|^{q} d x\right)^{1 / q} & =\left(\int\left|\omega_{0}^{\prime \prime \varepsilon}(x)\right|^{q}\right)^{1 / q} \\
& \leq\left\|\omega_{0}^{\prime \prime}\right\|_{L^{q}} \leq C_{q}\left\|\omega_{0}^{\prime \prime}\right\|_{L^{p}} \quad \forall q \in[1, p]
\end{aligned}
$$

(avec une constante $C_{q}$ indépendante de $t$ ).

Enfin, on remarquera que $v^{\varepsilon}$ est bornée dans l'espace $\operatorname{Lip}_{\text {loc }}\left(\mathbb{R}, \mathscr{D}^{\prime}\left(\mathbb{R}^{2} ; \mathbb{R}^{2}\right)\right)$ des fonctions localement lipschitziennes de $t$ à valeurs distributions: en effet, d'après de Lemma 1.1.2, (1.1.16) entraîne que

$$
\partial v^{\varepsilon} / \partial t=-\Delta^{-1} A(D)^{t}\left(\left(v_{1}^{\varepsilon}\right)^{2}-\left(v_{2}^{\varepsilon}\right)^{2}, v_{1}^{\varepsilon} \cdot v_{2}^{\varepsilon}\right)
$$

et on constate immédiatement que le membre de droite décrit lorsque $\varepsilon \in] 0,1$ ] et $t$ reste dans un intervalle compact une partie bornée de $\mathscr{D}^{\prime}\left(\mathbb{R}^{2} ; \mathbb{R}^{2}\right)$.

L'inégalité $(1.1 .17)$ entraîne qu'il existe $J \subset] 0,1]$ avec $0 \in \bar{J}$ et un élément $\check{v} \in L_{\text {loc }}^{\infty}\left(\mathbb{R}, L^{2}\left(\mathbb{R}^{2} ; \mathbb{R}^{2}\right)\right)$ tel que $\check{v}^{\varepsilon}$ converge, lorsque $\varepsilon$ tend vers 0 dans $J$, vers $\check{v}$ dans l'espace des distributions sur $\mathbb{R} \times \mathbb{R}^{2}$. On pose

$$
v(t, x)=\bar{v}(x)+\check{v}(t, x) .
$$

On a $v \in L_{\text {loc }}^{\infty}\left(\mathbb{R}, L_{\text {loc }}^{2}\left(\mathbb{R}^{2} ; \mathbb{R}^{2}\right)\right) \cap \operatorname{Lip}_{\text {loc }}\left(\mathbb{R}, \mathscr{D}^{\prime}\left(\mathbb{R}^{2} ; \mathbb{R}^{2}\right)\right)$.

D'après (1.1.19), $\omega^{\varepsilon}$ converge au sensdes distributions vers $\omega=\operatorname{rot}_{x} v$ 
lorsque $\varepsilon$ tend vers 0 dans $J$. D'autre part, la seconde inégalité (1.1.22) combinée à l'injection de $L^{q}\left(\mathbb{R}^{2}\right)$ dans $H^{-1}\left(\mathbb{R}^{2}\right)$ pour $\left.\left.q \in\right] 1,2\right]$ montre que $\omega^{\prime \prime}$ (et donc aussi $\left.\omega^{\ell}=\omega^{\varepsilon}-\omega^{\prime \ell}\right)$ est uniformément borné dans $L_{\mathrm{loc}}^{\infty}\left(\mathbb{R}, H^{-1}\left(\mathbb{R}^{2}\right)\right.$ ). Quitte à extraire de nouvelles sous-suites (que l'on notera de même), on peut donc supposer que $\omega^{\ell}$ et $\omega^{\prime \prime}$ convergent au sens des distributions, lorsque $\varepsilon$ tend vers 0 dans $J$, vers des limites $\omega^{\prime}$ et $\omega^{\prime \prime}$. On a $\omega=\omega^{\prime}+\omega^{\prime \prime}$, $\omega^{\prime}$ et $\omega^{\prime \prime}$ sont dans $L_{\text {loc }}^{\infty}\left(\mathbb{R}, H^{-1}\left(\mathbb{R}^{2}\right)\right)$ et d'après $(1.1 .22) \omega^{\prime}(t, \cdot)$ est pour presque tout $t \in \mathbb{R}$ une mesure positive de masse totale uniformément bornée et $\omega^{\prime \prime}$ est dans $L^{\infty}\left(\mathbb{R}, L^{q}\left(\mathbb{R}^{2}\right)\right)$ pour tout $q \in[1, p]$.

Supposons prouvée la proposition suivante,

Proposition 1.1.3. Les fonctions $v_{1}^{\varepsilon} \cdot v_{2}^{\varepsilon}\left(\right.$ resp. $\left.\left(v_{1}^{\varepsilon}\right)^{2}-\left(v_{2}^{\varepsilon}\right)^{2}\right)$ convergent au sens des distributions lorsque $\varepsilon$ tend vers 0 dans $J$ vers $v_{1} \cdot v_{2}\left(\right.$ resp. $\left.\left(v_{1}\right)^{2}-\left(v_{2}\right)^{2}\right)$; et montrons que le Théorème 1.1.1' en résulte.

D'après la Proposition 1.1.3 $V^{\varepsilon}={ }^{t}\left(\left(v_{1}^{\varepsilon}\right)^{2}-\left(v_{2}^{\varepsilon}\right)^{2}, v_{1}^{\varepsilon} \cdot v_{2}^{\varepsilon}\right)$ converge donc au sens des distributions vers $V={ }^{t}\left(v_{1}^{2}-v_{2}^{2}, v_{1} \cdot v_{2}\right)$. D'après le Lemme 1.1.2, pour tout $\varepsilon>0 \quad v^{\varepsilon}$ est solution de (1.1.8). Il suffit donc de voir que $\Delta^{-1} A(D) V^{\varepsilon}$ converge au sens des distributions vers $\Delta^{-1} A(D) V$ puisque alors $v$ est solution de (1.1.8) donc de (1.1.7). Or si $\varphi \in C_{0}^{\infty}\left(\mathbb{R} \times \mathbb{R}^{2} ; \mathbb{R}^{2}\right), \Delta^{-1 t} A(D) \varphi$ est $C^{\infty}$ sur $\mathbb{R} \times \mathbb{R}^{2}$ et est $O(1 /|x|)$ lorsque $x \rightarrow \infty$. D'après (1.1.17), $V^{\varepsilon}$ est somme de trois termes $V^{\varepsilon}=V_{1}^{\varepsilon}+V_{2}^{\varepsilon}+V_{3}$ où $V_{1}^{\varepsilon}$ est borné dans $L_{\text {loc }}^{\infty}\left(\mathbb{R}, L^{1}\left(\mathbb{R}^{2} ; \mathbb{R}^{2}\right)\right)$, $(1+|x|) V_{2}^{\varepsilon}$ est borné dans $L_{\text {loc }}^{\infty}\left(\mathbb{R}, L^{2}\left(\mathbb{R}^{2} ; \mathbb{R}^{2}\right)\right)$ et $\left|V_{3}\right|$ est $O\left(1 /\left(1+|x|^{2}\right)\right)$ dans $L^{\infty}$ et est indépendant de $t$. On en déduit immédiatement que $\left\langle V^{\varepsilon}, \Delta^{-1 t} A(D) \varphi\right\rangle$ converge vers $\left\langle V, \Delta^{-1 t} A(D) \varphi\right\rangle$.

La preuve du Théorème 1.1.1 est donc réduite à celle de la Proposition 1.1.3. Le paragraphe suivant lui est consacré.

Remarque. On remarquera que la pression $p$ correspondant à la solution $v$ du problème (1.1.6) n'est pas, en général, la limite des pressions $p^{\varepsilon}$ correspondant aux solutions $v^{\varepsilon}$ du problème à données régularisées (1.1.16): en effet, d'après (1.1.10), $p^{\varepsilon}$ dépend de tous les coefficients de la matrice $v^{\varepsilon} \otimes v^{\varepsilon}$ et pas seulement des quantités $v_{1}^{\varepsilon} \cdot v_{2}^{\varepsilon}$ et $\left(v_{1}^{\varepsilon}\right)^{2}-\left(v_{2}^{\varepsilon}\right)^{2}$ sur lesquelles on passe à la limite.

1.2. Passage à la limite. Dans ce paragraphe, fixons un réel $T>0$. Les diverses constantes utilisées pourront dépendre de $T$.

Théorème 1.2.1. Soit $\left.\left(\omega^{\varepsilon}\right)_{\varepsilon \in J}(J \subset] 0,1\right]$ avec $\left.0 \in \bar{J}\right)$ une famille de fonctions de $L^{\infty}\left([-T, T] ; C_{0}^{\infty}\left(\mathbb{R}^{2}\right)\right)$ vérifiant les conidtions suivantes:

(i) La famille des $\left(\omega^{\varepsilon}\right)_{\varepsilon \in J}$ est uniformément bornée dans

$$
\operatorname{Lip}\left([-T, T], \mathscr{D}^{\prime}\left(\mathbb{R}^{2}\right)\right) .
$$

(ii) Il existe $\omega^{\prime \varepsilon}$ et $\omega^{\prime \ell}$ dans $L^{\infty}\left([-T, T], C_{0}^{\infty}\left(\mathbb{R}^{2}\right)\right)$ avec $\omega^{\varepsilon}=\omega^{\ell}+$ $\omega^{\prime \prime \varepsilon}, \omega^{\prime \varepsilon} \geq 0,\left(\omega^{\prime \varepsilon}\right)_{\varepsilon \in J}\left(\right.$ resp. $\left.\left(\omega^{\prime \prime \varepsilon}\right)_{\varepsilon \in J}\right)$ bornée dans $L^{\infty}\left([-T, T], L^{1}\left(\mathbb{R}^{2}\right) \cap\right.$ $\left.H^{-1}\left(\mathbb{R}^{2}\right)\right)$ (resp. dans $L^{\infty}\left([-T, T], L^{q}\left(\mathbb{R}^{2}\right)\right)$ pour tout $q \in[1, p]$ avec $p>1$ 
fixé), $\omega^{\ell}$ (resp. $\left.\omega^{\prime \prime}\right)$ convergeant au sens des distributions vers $\omega^{\prime}$ (resp. $\left.\omega^{\prime \prime}\right)$ fonction $L^{\infty}$ de $t \in[-T, T]$ à valeurs dans les mesures positives ou nulles qui sont dans $H^{-1}\left(\mathbb{R}^{2}\right)$ (resp. élément de $L^{\infty}\left([-T, T], L^{q}\left(\mathbb{R}^{2}\right)\right.$ ) pour tout $q \in$ $[1, p])$.

(iii) Lorsque $\varepsilon$ tend vers 0 dans $J$, la suite $v^{\varepsilon}=\nabla^{\perp} \Delta^{-1} \omega^{\varepsilon}$ converge dans $\mathscr{D}^{\prime}\left([-T, T] \times \mathbb{R}^{2} ; \mathbb{R}^{2}\right)$ vers une limite $v$.

Alors la famille $v^{\varepsilon}$ est uniformément bornée dans $L^{\infty}\left([-T, T], L_{\mathrm{loc}}^{2}\left(\mathbb{R}^{2} ; \mathbb{R}^{2}\right)\right)$, sa limite $v$ est dans cet espace et lorsque $\varepsilon$ tend vers 0 dans $J$

$$
v_{1}^{\varepsilon} \cdot v_{2}^{\varepsilon} \rightarrow v_{1} \cdot v_{2}, \quad\left(v_{1}^{\varepsilon}\right)^{2}-\left(v_{2}^{\varepsilon}\right)^{2} \rightarrow\left(v_{1}\right)^{2}-\left(v_{2}\right)^{2}
$$

au sens des distributions sur $[-T, T] \times \mathbb{R}^{2}$.

On notera $\omega=\operatorname{rot} v=\lim _{\varepsilon \rightarrow 0, \varepsilon \in J} \omega^{\varepsilon}=\omega^{\prime}+\omega^{\prime \prime}$ : c'est une fonction $L^{\infty}$ de $t \in[-T, T]$ à valeurs dans les mesures finies qui sont dans $H^{-1}\left(\mathbb{R}^{2}\right)$ et une fonction lipschitzienne de $t$ à valeurs dans $\mathscr{D}^{\prime}\left(\mathbb{R}^{2}\right)$.

La borne uniforme de $v^{\varepsilon}$ dans $L^{\infty}\left([-T, T], L_{\text {loc }}^{2}\left(\mathbb{R}^{2} ; \mathbb{R}^{2}\right)\right)$ dans l'énonce précédent est immédiate. Il nous faut prouver ici que (1.2.1) a lieu. Il sous suffit d'ailleurs de montrer la première de ces relations puisqu'on peut toujours écrire $\left(v_{1}^{\varepsilon}\right)^{2}-\left(v_{2}^{\varepsilon}\right)^{2}=\left(v_{1}^{\varepsilon}-v_{2}^{\varepsilon}\right)\left(v_{1}^{\varepsilon}+v_{2}^{\varepsilon}\right)$ et se ramener à la première forme par rotation des axes de coordonnées.

Nous utiliserons dans le cours de la preuve le lemme immédiat suivant, qui résulte de l'hypothèse (i) précédente.

Lemme 1.2.2. Si $\left(t_{k}\right)_{k \in \mathbb{N}}$ est une suite de points de $[-T, T]$ convergeant vers $t_{\infty}$ et si $\left(\varepsilon_{k}\right)_{k \in \mathbb{N}}$ est une suite de points de $J$ convergeant vers 0 , la suite $\omega^{\varepsilon_{k}}\left(t_{k}, \cdot\right)$ converge dans $\mathscr{D}^{\prime}\left(\mathbb{R}^{2}\right)$ vers $\omega\left(t_{\infty}, \cdot\right)$.

Soient $\varphi \in C_{0}^{\infty}\left(\mathbb{R}^{2}\right), \psi \in C^{\infty}([-T, T])$. Il nous faut, pour montrer le théorème, prouver que

$$
\begin{aligned}
\int_{-T}^{T} \int & v_{1}^{\varepsilon}(t, z) v_{2}^{\varepsilon}(t, z) \varphi(z) \psi(t) d z d t \\
& \rightarrow \int_{-T}^{T} \int v_{1}(t, z) v_{2}(t, z) \varphi(z) \psi(t) d z d t
\end{aligned}
$$

lorsque $\varepsilon$ tend vers 0 dans $J$. En écrivant $v^{\varepsilon}=\nabla^{\perp} \Delta^{-1} \omega^{\varepsilon}$ et d'après (1.1.5), le membre de gauche de (1.2.2) n'est autre que

$$
\int_{-T}^{T} \int \omega^{\varepsilon}(t, x) \omega^{\varepsilon}(t, y) \psi(t) H_{\varphi}(x, y) d x d y d t,
$$

où $H_{\varphi}$ est la distribution sur $\mathbb{R}^{2} \times \mathbb{R}^{2}$

$$
H_{\varphi}(x, y)=-\frac{1}{4 \pi^{2}} \frac{\partial^{2}}{\partial x_{1} \partial y_{2}} \int \log |x-z| \log |y-z| \varphi(z) d z .
$$


La preuve du théorème repose sur la description de la distribution $H_{\varphi}$ fournie par la proposition suivante:

Proposition 1.2.3. La distribution $H_{\varphi}$ est une fonction bornée sur $\mathbb{R}^{2} \times \mathbb{R}^{2}$, continue sur le complémentaire de la diagonale, tendant vers 0 à l'infini. De plus, on peut écrire

$$
H_{\varphi}(x, y)=\frac{1}{2}(\varphi(x)+\varphi(y)) h(x-y)+r(x, y),
$$

où $r$ est une fonction continue bornée sur $\mathbb{R}^{2} \times \mathbb{R}^{2}$ et h est la fonction homogène de degré 0

$$
h(w)=\frac{1}{4 \pi} \frac{w_{1} \cdot w_{2}}{|w|^{2}} .
$$

La restriction de $H_{\varphi}$ au complémentaire de la diagonale s'écrit

$$
-\frac{1}{4 \pi^{2}} W_{\varphi}(y, x-y),
$$

où $W_{\varphi}(y, w)$ est la fonction définie sur $\mathbb{R}^{2} \times\left(\mathbb{R}^{2} \backslash\{0\}\right)$ par

$$
W_{\varphi}(y, w)=\int \frac{z_{2}}{|z|^{2}} \frac{w_{1}+z_{1}}{|w+z|^{2}} \varphi(y-z) d z .
$$

En particulier $W_{\varphi}$ est continue sur $\mathbb{R}^{2} \times\left(\mathbb{R}^{2} \backslash\{0\}\right)$ et $\lim _{(y, w) \rightarrow \infty, w \neq 0} W_{\varphi}(y, w)$ $=0$. Prouvons le lemme suivant:

Lemme 1.2.4. La fonction définie sur $\mathbb{R}^{2} \times\left(\mathbb{R}^{2} \backslash\{0\}\right) \quad(y, w) \rightarrow W_{\varphi}(y, w)+$ $4 \pi^{2} \varphi(y) h(w)$ se prolonge continument à $\mathbb{R}^{2} \times \mathbb{R}^{2}$.

Démonstration. Soit $K$ un compact de $\mathbb{R}^{2}$. Choisissons une fonction $\theta \in$ $C_{0}^{\infty}\left(\mathbb{R}^{2}\right)$ radiale égale à 1 sur une boule voisinage de $\left\{y-y^{\prime} ; y \in K, y^{\prime} \in\right.$ $\operatorname{Supp} \varphi\}$. Lorsque $y$ reste dans $K$ et $y-z$ est voisin de $\operatorname{Supp} \varphi$ on a

$$
\varphi(y-z)=\varphi(y) \theta(z)+s(y, z),
$$

où $s(y, z)$ est $C^{\infty}$ à support compact en $(y, z)$ et vérifie $|s(y, z)| \leq \operatorname{cst}|z|$ pour tout $(y, z) \in \mathbb{R}^{2} \times \mathbb{R}^{2}$.

On a alors pour $y \in K, w \in \mathbb{R}^{2} \backslash\{0\}$ d'après (1.2.8)

$$
W_{\varphi}(y, w)=\varphi(y) W(w)+\int \frac{z_{2}}{|z|^{2}} \frac{w_{1}+z_{1}}{|w+z|^{2}} s(y, z) d z,
$$

où

$$
W(w)=\int \frac{z_{2}}{|z|^{2}} \frac{w_{1}+z_{1}}{|w+z|^{2}} \theta(z) d z .
$$

Comme $s(y, 0)=0$ on voit, par le théorème de convergence dominée, que le second terme du membre de droite de (1.2.10) définit une fonction continue sur 
$K \times \mathbb{R}^{2}$. Il nous faut donc voir, pour prouver le lemme, que $W(w)+4 \pi^{2} h(w)$ se prolonge continûment à $\mathbb{R}^{2}$. Il suffit pour cela de montrer que $W(\sigma w)+$ $4 \pi^{2} h(w)$ tend vers 0 uniformément en $w$ décrivant la sphère de centre 0 de rayon 1 lorsque $\sigma$ tend vers $0+$. Or, si $0<\sigma^{\prime}<\sigma<1$ on a

$$
W(\sigma w)-W\left(\sigma^{\prime} w\right)=\int \frac{z_{2}}{|z|^{2}} \frac{w_{1}+z_{1}}{|w+z|^{2}}\left(\theta(\sigma z)-\theta\left(\sigma^{\prime} z\right)\right) d z,
$$

où l'intégrant est à support dans une couronne $C_{1} / \sigma \leq|z| \leq C_{2} / \sigma^{\prime}$. Comme $\theta$ est radiale et que $z_{1} z_{2} /|z|^{4}$ est de moyenne nulle sur la sphère unité, cette expression vaut aussi

$$
\int \frac{z_{2}}{|z|^{2}}\left(\frac{w_{1}+z_{1}}{|w+z|^{2}}-\frac{z_{1}}{|z|^{2}}\right)\left(\theta(\sigma z)-\theta\left(\sigma^{\prime} z\right)\right) d z
$$

et donc se majore en module par Cst $\int_{C_{1}<\sigma|z|} d z /|z|^{3}$ qui tend vers 0 si $\sigma \rightarrow 0+$.

Il en résulte donc que $W$ est localement bornée au voisinage de 0 , admet des limites radiales en 0 et que la suite des $W_{\sigma}(\cdot)=W(\sigma \cdot)$ converge au sens des distributions lorsque $\sigma$ tend vers $0+$ vers la fonction homogène de degré 0 donnée par ces limites radiales. Pour calculer cette limite, remarquons que puisque

$$
W(\sigma w)=\int \frac{z_{2}}{|z|^{2}} \frac{w_{1}+z_{1}}{|w+z|^{2}} \theta(\sigma z) d z, \quad w \in \mathbb{R}^{2} \backslash\{0\},
$$

et que la transformée de Fourier de $z_{1} /|z|^{2}$ est $-2 i \pi \zeta_{1} /|\zeta|^{2}$, la transformée de Fourier de $W_{\sigma}$ est

$$
-2 i \pi \frac{\zeta_{1}}{|\zeta|^{2}} \int e^{i z \cdot \zeta} \frac{z_{2}}{|z|^{2}} \theta(\sigma z) d z
$$

Si $g \in C_{0}^{\infty}\left(\mathbb{R}^{2}\right)$ vérifie $g(0)=0$, on a alors

$$
\begin{aligned}
\lim _{\sigma \rightarrow 0+}\left\langle\widehat{W}_{\sigma}, g\right\rangle & =-2 i \pi \lim _{\sigma \rightarrow 0+} \int e^{i z \cdot \zeta} \frac{z_{2}}{|z|^{2}} \theta(\sigma z) \frac{\zeta_{1} g(\zeta)}{|\zeta|^{2}} d z d \zeta \\
& =\lim _{\sigma \rightarrow 0+} \int \frac{\zeta_{2}+\sigma \zeta_{2}^{\prime}}{\left|\zeta+\sigma \zeta^{\prime}\right|^{2}} \hat{\theta}\left(\zeta^{\prime}\right) \frac{\zeta_{1} g(\zeta)}{|\zeta|^{2}} d \zeta d \zeta^{\prime} \\
& =\lim _{\sigma \rightarrow 0+} \int \frac{\zeta_{2}}{|\zeta|^{2}} \hat{\theta}\left(\zeta^{\prime}\right) \frac{\left(\zeta_{1}-\sigma \zeta_{1}^{\prime}\right) g\left(\zeta-\sigma \zeta^{\prime}\right)}{\left|\zeta-\sigma \zeta^{\prime}\right|^{2}} d \zeta d \zeta^{\prime} \\
& =4 \pi^{2} \int \frac{\zeta_{1} \zeta_{2}}{|\zeta|^{4}} g(\zeta) d \zeta .
\end{aligned}
$$

Si maintenant $g$ est une fonction quelconque de $C_{0}^{\infty}\left(\mathbb{R}^{2}\right)$, choisissons $\beta \in$ $C_{0}^{\infty}\left(\mathbb{R}^{2}\right)$ radiale, égale à 1 près de 0 et remarquons que

$$
\left\langle\widehat{W}_{\sigma}, \beta\right\rangle=-2 i \pi \int e^{i z \cdot \zeta} \frac{z_{2}}{|z|^{2}} \theta(\sigma z) \frac{\zeta_{1} \beta(\zeta)}{|\zeta|^{2}} d z d \zeta=0
$$


comme on le voit en changeant $\left(z_{1}, \zeta_{1}\right)$ en $\left(-z_{1}-\zeta_{1}\right)$ et un utilisant que $\theta$ et $\beta$ sont radiales. On voit donc que

$$
\begin{aligned}
\lim _{\sigma \rightarrow 0+}\left\langle\widehat{W}_{\sigma}, g\right\rangle & =4 \pi^{2} \int \frac{\zeta_{1} \zeta_{2}}{|\zeta|^{4}}(g(\zeta)-g(0) \beta(\zeta)) d \zeta \\
& =\lim _{\delta \rightarrow 0+} 4 \pi^{2} \int_{|\zeta| \geq \delta} \frac{\zeta_{1} \zeta_{2}}{|\zeta|^{4}} g(\zeta) d \zeta
\end{aligned}
$$

Lorsque $\sigma \rightarrow 0+, W_{\sigma}$ converge donc vers la transformée de Fourier inverse de la distribution définie par le membre de droite de (1.2.18), c'est-à-dire, vers $-\pi w_{1} w_{2} /|w|^{2}=-4 \pi^{2} h(w)$. Cela achève la preuve du lemme.

Fin de la preuve de la Proposition 1.2.3. D'après le Lemme 1.2.4, $W_{\varphi}(y, w)$ définit une fonction bornée sur $\mathbb{R}^{2} \times \mathbb{R}^{2}$, continue hors de $w=0$, tendant vers 0 à l'infini et d'après (1.2.7) la distribution $H_{\varphi}$ ne differe donc du membre de droite de (1.2.5) que par une distribution portée par la diagonale $x=y$. Or, par définition, si $f \in C_{0}^{\infty}\left(\mathbb{R}^{4}\right)$

$$
\left\langle H_{\varphi}, f\right\rangle=-\frac{1}{4 \pi^{2}} \int \frac{y_{2}-z_{2}}{|y-z|^{2}} \frac{x_{1}-z_{1}}{|x-z|^{2}} \varphi(z) f(x, y) d x d y d z
$$

et comme le noyau est localement intégrable sur $\mathbb{R}^{4}$, il est immédiat que $H_{\varphi}$ est d'ordre 0 et ne charge pas de sous-variété. L'égalité (1.2.5) est donc vraie.

Nous allons maintenant utiliser la Proposition 1.2.3 pour prouver le Théorème 1.2.1. Nous nous servirons du lemme suivant:

Lemme 1.2.5. Soit $\alpha$ une mesure finie qui est dans l'espace de Sobolev $H^{-1}\left(\mathbb{R}^{2}\right)$. Alors $\alpha$ est diffuse (i.e. $\forall x_{0} \in \mathbb{R}^{2} \alpha\left(\left\{x_{0}\right\}\right)=0$ ).

Démonstration. Toute mesure finie se décompose de manière unique en $\alpha=$ $\alpha_{d}+\alpha_{a}$ où $\alpha_{d}$ est diffuse et où $\alpha_{a}$ est atomique, i.e., $\alpha_{a}=\sum_{1}^{+\infty} \lambda_{j} \delta_{x_{j}}$ où $\left(x_{j}\right)_{j \in \mathbb{N}^{*}}$ est une suite de $\mathbb{R}^{2}$ et $\left(\lambda_{j}\right)_{j \in \mathbb{N}^{*}}$ est une suite dans $l^{1}$. Soit $x_{0}$ un point de $\mathbb{R}^{2}$ et si $g \in C_{0}^{\infty}\left(\mathbb{R}^{2}\right)$ vérifie $g \equiv 1$ près de 0 , posons pour tout $\left.\left.\sigma \in\right] 0,1\right]$ $g_{\sigma}(x)=g\left(\left(x-x_{0}\right) / \sigma\right)$. Alors la famille $\left(g_{\sigma}\right)_{\sigma \in] 0,1]}$ est bornée dans $H^{1}\left(\mathbb{R}^{2}\right)$ et tend faiblement vers 0 dans $H^{1}$. Puisque $\alpha \in H^{-1}, \lim _{\sigma \rightarrow 0+}\left\langle\alpha, g_{\sigma}\right\rangle=0$. Or, par convergence dominée $\left\langle\alpha_{d}, g_{\sigma}\right\rangle \rightarrow 0$ (puisque $g_{\sigma} \rightarrow 0 \alpha_{d}$-presque partout). D'autre part, $\left\langle\alpha_{a}, g_{\sigma}\right\rangle$ tend vers 0 si $x_{0} \neq x_{j} \forall j \in \mathbb{N}^{*}$ et vers $\lambda_{j}$ s'il existe $j \in \mathbb{N}^{*}$ avec $x_{0}=x_{j}$. On en déduit donc $\lambda_{j}=0$ pour tout $j$, i.e., $\alpha=\alpha_{d}$.

Pour tout $t \in[-T, T]$ on notera $\omega_{t}$ la limite des $\omega^{\varepsilon}(t, \cdot)$ (qui existe d'après le Lemme 1.2.2). Comme d'après l'hypothèse (ii) du Théorème $1.2 .1 \omega^{\varepsilon}(t, \cdot)$ est bornée dans $H^{-1}\left(\mathbb{R}^{2}\right) \cap L^{1}\left(\mathbb{R}^{2}\right)$ (en utilisant l'injection $L^{q}\left(\mathbb{R}^{2}\right) \subset H^{-1}\left(\mathbb{R}^{2}\right)$ pour $q \in] 1,2]), \omega_{t}$ est une mesure finie qui est dans $H^{-1}\left(\mathbb{R}^{2}\right)$ et donc, d'après le Lemme 1.2.5, qui est diffuse. Sa variation totale $\left|\omega_{t}\right|$ est donc également 
diffuse. Cela entraîne que la diagonale $x=y$ de $\mathbb{R}^{2} \times \mathbb{R}^{2}$ est de $d\left|\omega_{t}\right|(x) \otimes$ $d\left|\omega_{t}\right|(y)$-mesure nulle. En effet, si $\mathbf{1}_{\{x=y\}}$ est la fonction caractéristique de la diagonale

$$
\begin{aligned}
\int \mathbf{1}_{\{x=y\}} d\left|\omega_{t}\right|(x) \otimes d\left|\omega_{t}\right|(y) & =\int d\left|\omega_{t}\right|(y)\left(\int \mathbf{1}_{\{x=y\}} d\left|\omega_{t}\right|(x)\right) \\
& =\int\left|\omega_{t}\right|(\{y\}) d\left|\omega_{t}\right|(y)
\end{aligned}
$$

et cette dernière quantité est nulle puisque l'intégrant est nul pour tout $y \in \mathbb{R}^{2}$.

La fonction $H_{\varphi}$, qui d'après la Proposition 1.2.3 est bornée sur $\mathbb{R}^{2} \times \mathbb{R}^{2}$ et continue hors de la diagonale, définit donc sans ambiguité un élément de l'espace $L^{\infty}\left(\mathbb{R}^{2} \times \mathbb{R}^{2} ; d \omega_{t}(x) \otimes d \omega_{t}(y)\right)$ des fonctions mesurables essentiellement bornées pour la mesure $d \omega_{t}(x) \otimes d \omega_{t}(y)$. Si $\psi \in C^{\infty}([-T, T])$ on peut donc définir l'intégrale

$$
\int_{-T}^{T} \int H_{\varphi}(x, y) \psi(t) d \omega_{t}(x) \otimes d \omega_{t}(y) d t .
$$

Nous allons prouver la proposition suivante:

Proposition 1.2.6. Soit $\left(\omega^{\varepsilon}\right)_{\varepsilon \in J}$ une famille de fonctions vérifiant les hypothèses (i) et (ii) $d u$ Théorème 1.2.1.

Alors pour toutes fonctions $\varphi \in C_{0}^{\infty}\left(\mathbb{R}^{2}\right), \psi \in C^{\infty}([-T, T])$

$$
\begin{gathered}
\lim _{\substack{\varepsilon \rightarrow 0 \\
\varepsilon \in J}} \int H_{\varphi}(x, y) \psi(t) \omega^{\varepsilon}(t, x) \omega^{\varepsilon}(t, y) d x d y d t \\
=\int H_{\varphi}(x, y) \psi(t) d \omega_{t}(x) \otimes d \omega_{t}(y) d t .
\end{gathered}
$$

Démonstration. Soit $\chi \in C_{0}^{\infty}\left(\mathbb{R}^{2}\right)$ vérifiant $\chi \equiv 1$ au voisinage de 0 . L'expression obtenue en substituant dans le membre de gauche de (1.2.22) la fonction

$$
[(1-\chi) h](x-y)\left(\frac{\varphi(x)+\varphi(y)}{2}\right)+r(x, y)
$$

à $H_{\varphi}(x, y)$ converge vers

$$
\int\left([(1-\chi) h](x-y)\left(\frac{\varphi(x)+\varphi(y)}{2}\right)+r(x, y)\right) \psi(t) d \omega_{t}(x) \otimes d \omega_{t}(y) d t
$$

puisque d'après la Proposition 1.2.3, (1.2.23) est une fonction continue tendant vers 0 à l'infini et que $\omega^{\varepsilon}$ converge faiblement vers $\omega$ en restant bornée dans $L^{\infty}\left([-T, T], L^{1}\left(\mathbb{R}^{2}\right)\right)$. Il nous suffit donc de voir que

$$
\begin{gathered}
\lim _{\substack{\varepsilon \rightarrow 0 \\
\varepsilon \in J}} \int \chi h(x-y) \varphi(y) \psi(t) \omega^{\varepsilon}(t, x) \omega^{\varepsilon}(t, y) d x d y d t \\
=\int \chi h(x-y) \varphi(y) \psi(t) d \omega_{t}(x) d \omega_{t}(y) d t
\end{gathered}
$$

(et la même égalité avec $\varphi(y)$ remplacée par $\varphi(x)$ ). 
Pour cela choisissons $R_{0}$ assez grand pour que le support de $(x, y) \rightarrow$ $\chi h(x-y) \varphi(y)$ soit contenu dans le produit des boules $B\left(0, R_{0}\right) \times B\left(0, R_{0}\right)$ et prouvons le lemme suivant, dont une version analogue, mais plus précise, a été obtenue par Majda [16, formule (2.9)].

Lemme 1.2.7. Il existe une famille $\left(V_{j}\right)_{j \in \mathbb{N}^{*}}$ de voisinages ouverts de la diagonale de $\overline{B\left(0, R_{0}\right)} \times \overline{B\left(0, R_{0}\right)}$ et une suite $\left(\varepsilon_{j}\right)_{j \in \mathbb{N}^{*}}$ de $\left.] 0,1\right]$ tels que

$$
\left.\left.\int_{-T}^{T} \int_{V_{j}}\left|\omega^{\varepsilon}(t, x) \| \omega^{\varepsilon}(t, y)\right| d x d y \leq \frac{1}{j} \quad \text { pour tout } \varepsilon \in\right] 0, \varepsilon_{j}\right] .
$$

Démonstration. Montrons qu'il existe une suite $\left(\varepsilon_{j}\right)_{j \in \mathbb{N}^{*}}$ et une suite $\left(r_{j}\right)_{j \in \mathbb{N}^{*}}$ tendant vers 0 dans $] 0,1]$ telles que $(1.2 .26)$ soit vérifiée avec

$$
V_{j}=\left\{(x, y) \in \overline{B\left(0, R_{0}\right)} \times \overline{B\left(0, R_{0}\right)} ;|x-y| \leq r_{j}\right\} .
$$

En effet, le membre de gauche de (1.2.26) se majore par

$$
\left(\sup _{t \in[-T, T]} \sup _{x \in \overline{B\left(0, R_{0}\right)}} \int_{|z| \leq r_{j}}\left|\omega^{\varepsilon}(t, x+z)\right| d z\right)\left(\int_{-T}^{T} \int\left|\omega^{\varepsilon}(t, x)\right| d x d t\right)
$$

et le second facteur est uniformément majoré en $\varepsilon$ par une constante $C$ d'après (1.1.22). Il nous suffit de voir que les suites $\left(\varepsilon_{j}\right)_{j \in \mathbb{N}^{*}},\left(r_{j}\right)_{j \in \mathbb{N}^{*}}$ peuvent être choisies de telle manière que le premier se majore par $1 / C j$ pour tout $\varepsilon \in$ ]0, $\left.\varepsilon_{j}\right]$. Si ce n'est pas le cas, il existe $\delta>0$ et pout tout $\left.N, \varepsilon_{N} \in\right] 0,1 / N[$, $x_{N} \in \overline{B\left(0, R_{0}\right)}$ tels que

$$
\delta \leq \sup _{t \in[-T, T]} \int_{|z| \leq 1 / N}\left|\omega^{\varepsilon_{N}}\left(t, x_{N}+z\right)\right| d z .
$$

Quitte à diminuer $\delta$, il existe pour tout $N, t_{N} \in[-T, T]$ tel que

$$
\left\|\omega^{\prime \prime \varepsilon_{N}}\left(t_{N}, \cdot\right)\right\|_{L^{p}} \leq\left\|\omega^{\prime \prime \varepsilon_{N}}\right\|_{L^{\infty}\left([-T, T], L^{p}\right)}
$$

et que

$$
\delta \leq \int_{|z| \leq 1 / N}\left|\omega^{\varepsilon_{N}}\left(t_{N}, x_{N}+z\right)\right| d z .
$$

Quitte à extraire une sous-suite, on peut supposer que $x_{N}$ converge vers un point $x_{0}$ de $\overline{B\left(0, R_{0}\right)}$ et que $t_{N}$ converge vers un point $t_{0}$ de $[-T, T]$. Soit $g \in C_{0}^{\infty}\left(\mathbb{R}^{2}\right), g \geq 0, g \equiv 1$ près de $x_{0}$. D'après la positivité de $\omega^{\ell}$, on a

$$
\begin{aligned}
& \int \omega^{\varepsilon_{N}}\left(t_{N}, x\right) g(x) d x \\
& \quad \geq \int\left|\omega^{\varepsilon_{N}}\left(t_{N}, x\right)\right| g(x) d x-2 \int\left|\omega^{\prime \varepsilon_{N}}\left(t_{N}, x\right)\right| g(x) d x .
\end{aligned}
$$

D'après (1.2.29) et les inégalités (1.1.22), le membre de gauche de (1.2.30) se minore donc pour $N$ assez grand par

$$
\delta-C_{p}\left\|\omega_{0}^{\prime \prime}\right\|_{L^{p}}\|g\|_{L^{q}}
$$

$q$ désignant l'exposant conjugué de $p$. 
On voit, d'après le Lemme 1.2.2 que

$$
\int g(x) d \omega_{t_{0}}(x) \geq \delta-C_{p}\left\|\omega_{0}^{\prime \prime}\right\|_{L^{p}}\|g\|_{L^{q}} .
$$

Comme $q<+\infty$ une telle inégalité est contradictoire avec le fait que, d'après le Lemme 1.2.5, $\omega_{t_{0}}$ est diffuse.

Fin de la preuve de la Proposition 1.2.6. Il nous faut prouver (1.2.25). Soit $\eta>0$ fixé. Pour tout $j$ choisissons une fonction $\theta_{j} \in C_{0}^{\infty}\left(\mathbb{R}^{2}\right)$ à valeurs dans $[0,1], \theta_{j} \equiv 1$ près de 0 , telle que $\operatorname{Supp} \theta_{j}(x-y) \cap\left(B\left(0, R_{0}\right) \times B\left(0, R_{0}\right)\right)$ soit contenu dans $V_{j}$. Puisque la diagonale est de $d \omega_{t}(x) \otimes d \omega_{t}(y)$-mesure nulle pour tout $t \in[-T, T], \chi h(x-y) \varphi(y)\left(1-\theta_{j}(x-y)\right)$ converge vers $\chi h(x-y) \varphi(y) d \omega_{t}(x) \otimes d \omega_{t}(y)$-presque partout lorsque $j$ tend vers l'infini et donc, par convergence dominée

$$
\begin{aligned}
& \mid \int_{-T}^{T} \int \chi h(x-y) \varphi(y)\left(1-\theta_{j}(x-y)\right) \psi(t) d \omega_{t}(x) \otimes d \omega_{t}(y) d t \\
& \quad-\int_{-T}^{T} \int \chi h(x-y) \varphi(y) \psi(t) d \omega_{t}(x) \otimes d \omega_{t}(y) d t \mid<\frac{\eta}{3}
\end{aligned}
$$

si $j \geq j_{1}$ assez grand. D’autre part

$$
\begin{aligned}
& \left|\int_{-T}^{T} \int \chi h(x-y) \varphi(y) \theta_{j}(x-y) \psi(t) \omega^{\varepsilon}(t, x) \omega^{\varepsilon}(t, y) d x d y d t\right| \\
& \quad \leq\|\chi h\|_{L^{\infty}}\|\varphi\|_{L^{\infty}}\|\psi\|_{L^{\infty}} \int_{-T}^{T} \int_{V_{j}}\left|\omega^{\varepsilon}(t, x) \| \omega^{\varepsilon}(t, y)\right| d x d y d t
\end{aligned}
$$

qui s'estime, d'après le Lemme 1.2.7, par $\eta / 3$ lorsque $j$ est fixé assez grand et que $\varepsilon$ décrit $] 0, \varepsilon_{j}[$.

Enfin la fonction $\chi h(x-y) \varphi(y)\left(1-\theta_{j}(x-y)\right)$ étant continue, pour tout $\varepsilon \in J$ assez petit

$$
\begin{aligned}
& \mid \int_{-T}^{T} \int \chi h(x-y)\left(1-\theta_{j}(x-y)\right) \varphi(y) \psi(t) \omega^{\varepsilon}(t, x) \omega^{\varepsilon}(t, y) d x d y d t \\
& \quad-\int_{-T}^{T} \int \chi h(x-y)\left(1-\theta_{j}(x-y)\right) \varphi(y) \psi(t) d \omega_{t}(x) \otimes d \omega_{t}(y) d t \mid<\frac{\eta}{3}
\end{aligned}
$$

par convergence faible de $\omega^{\varepsilon}(t, x) \otimes \omega^{\varepsilon}(t, y)$ vers $\omega_{t}(x) \otimes \omega_{t}(y)$ pour tout $t \in[-T, T]$. Il résulte donc de (1.2.33), (1.2.34), (1.2.35) que pour $\varepsilon \in J$ assez petit

$$
\begin{aligned}
\mid \int_{-T}^{T} \int \chi h(x-y) \varphi(y) \psi(t) \omega^{\varepsilon}(t, x) \omega^{\varepsilon}(t, y) d x d y d t \\
\quad-\int_{-T}^{T} \int \chi h(x-y) \varphi(y) \psi(t) d \omega_{t}(x) \otimes d \omega_{t}(y) d t \mid<\eta
\end{aligned}
$$

ce qui conclut la preuve de la Proposition 1.2.6. 
Remarque. On notera que l'on n'a pas utilisé dans la preuve la description explicite de $H_{\varphi}$ fournie par la Proposition 1.2.3. Seul importe le fait que $H_{\varphi}$ soit une fonction bornée, tendant vers 0 à l'infini, continue hors de la diagonale.

Fin de la preuve du Théorème 1.2.1. D'après la Proposition 1.2.6 et (1.2.3) on a

$$
\begin{aligned}
\lim _{\substack{\varepsilon \rightarrow 0 \\
\varepsilon \in J}} \int_{-T}^{T} \int v_{1}^{\varepsilon}(t, z) v_{2}^{\varepsilon}(t, z) \varphi(z) \psi(t) d z d t \\
\quad=\int_{-T}^{T} \int H_{\varphi}(x, y) \psi(t) d \omega_{t}(x) d \omega_{t}(y) d t
\end{aligned}
$$

et il nous suffit de montrer que le membre de droite de (1.2.37) n'est autre que

$$
\int_{-T}^{T} \int v_{1}(t, z) v_{2}(t, z) \varphi(z) \psi(t) d z d t .
$$

Pour cela choisissons $\rho_{j} \in C_{0}^{\infty}\left(\mathbb{R}^{2}\right), \rho_{j} \geq 0, j=1,2$, vérifiant $\int \rho_{1} d x \equiv 1$, $\rho_{2} \equiv 1$ près de 0 et avec la notation $\rho_{1}^{\varepsilon}(x)=\left(1 / \varepsilon^{2}\right) \rho_{1}(x / \varepsilon)$ définissons:

$$
\begin{aligned}
\underline{\omega}^{\varepsilon}(t, x) & =\rho_{1}^{\varepsilon} *\left(\rho_{2}(\varepsilon \cdot) \omega(t, \cdot)\right), \\
\underline{\omega}^{\prime} & (t, x)=\rho_{1}^{\varepsilon} *\left(\rho_{2}(\varepsilon \cdot) \omega^{\prime}(t, \cdot)\right), \\
\underline{\omega}^{\prime \prime} & (t, x)=\rho_{1}^{\varepsilon} *\left(\rho_{2}(\varepsilon \cdot) \omega^{\prime \prime}(t, \cdot)\right) .
\end{aligned}
$$

Alors, les suites $\underline{\omega}^{\varepsilon}, \underline{\omega}^{\prime}, \underline{\omega}^{\prime \ell}$ vérifient les hypothèses (i) et (ii) du Théorème 1.2.1. De plus, pour tout $t$ fixé, $\underline{\omega}^{\varepsilon}(t, \cdot)$ converge vers $\omega(t, \cdot)$ fortement dans $H^{-1}\left(\mathbb{R}^{2}\right)$. Si l'on pose $\underline{v}^{\varepsilon}(t, \cdot)=\nabla^{\perp} \Delta^{-1} \underline{\omega}^{\varepsilon}(t, \cdot)$, on en déduit aisément que lorsque $\varepsilon \rightarrow 0, \underline{v}^{\varepsilon}(t, \cdot)$ converge fortement dans $L_{\text {loc }}^{2}\left(\mathbb{R}^{2} ; \mathbb{R}^{2}\right)$ vers $v(t, \cdot)=\nabla^{\perp} \Delta^{-1} \omega(t, \cdot)$ pour tout $t$ fixé. Comme de plus $\underline{v}^{\varepsilon}$ est bornée dans $L^{\infty}\left([-T, T], L_{\text {loc }}^{2}\left(\mathbb{R}^{2} ; \mathbb{R}^{2}\right)\right)$ il en résulte

$$
\begin{aligned}
\int_{-T}^{T} & \int v_{1}(t, z) v_{2}(t, z) \varphi(z) \psi(t) d z d t \\
\quad= & \lim _{\varepsilon \rightarrow 0} \int_{-T}^{T} \int \underline{v}_{1}^{\varepsilon}(t, z) \underline{v}_{2}^{\varepsilon}(t, z) \varphi(z) \psi(t) d z d t .
\end{aligned}
$$

Mais d'après (1.2.3), l'intégrale du membre de droite s'écrit

$$
\int_{-T}^{T} \int \underline{\omega}^{\varepsilon}(t, x) \underline{\omega}^{\varepsilon}(t, y) H_{\varphi}(x, y) \psi(t) d x d y d t
$$

et comme les suites $\underline{\omega}^{\varepsilon}, \underline{\omega}^{\ell}, \underline{\omega}^{\prime \prime}$ vérifient les hypothèses de la Proposition 1.2.6, le membre de droite de (1.2.40) n'est autre que

$$
\int_{-T}^{T} \int H_{\varphi}(x, y) \psi(t) d \omega_{t}(x) \otimes d \omega_{t}(y) d t .
$$

La relation (1.2.2) découle alors de (1.2.37) et de (1.2.40). Cela achève la preuve du Théorème 1.2.1. 
Remarque. On peut, pour prouver le Théorème 1.1.1, procéder de même mais en choisissant un autre type d'approximations $\omega^{\varepsilon}$. Par exemple, on peut définir $\omega_{0}^{\varepsilon}, v_{0}^{\varepsilon}$ comme en (1.1.13), (1.1.15) et ensuite prendre pour $v^{\varepsilon}$ sur l'intervalle $[0,+\infty[$ la solution des équations de Navier-Stokes

$$
\left\{\begin{array}{l}
\partial v^{\varepsilon} / \partial t+\left(v^{\varepsilon} \cdot \nabla\right) v^{\varepsilon}=-\nabla p^{\varepsilon}+\varepsilon \Delta v^{\varepsilon}, \\
\operatorname{div} v^{\varepsilon}=0 \\
\left.v^{\varepsilon}\right|_{t=0}=v_{0}^{\varepsilon}
\end{array}\right.
$$

Le tourbillon $\omega^{\varepsilon}$ correspondant vérifie alors

$$
\left\{\begin{array}{l}
\partial \omega^{\varepsilon} / \partial t+\left(v^{\varepsilon} \cdot \nabla\right) \omega^{\varepsilon}=\varepsilon \Delta \omega^{\varepsilon}, \\
\left.\omega^{\varepsilon}\right|_{t=0}=\omega_{0}^{\varepsilon} .
\end{array}\right.
$$

On définit $\omega^{\prime \varepsilon}$ et $\omega^{\prime \prime}$ comme les solutions de la première équation (1.2.44) avec les données $\omega_{0}^{\prime \varepsilon}$ et $\omega_{0}^{\prime \prime}$ respectivement. En outre, si $\bar{\omega} \in C_{0}^{\infty}\left(\mathbb{R}^{2}\right)$ est radiale et telle que $\int \bar{\omega}=\int \omega_{0}$, on note $\bar{\omega}^{\varepsilon}(t, x)$ la solution de

$$
\left\{\begin{array}{l}
\partial \bar{\omega}^{\varepsilon} / \partial t=\varepsilon \Delta \bar{\omega}^{\varepsilon}, \\
\left.\bar{\omega}^{\varepsilon}\right|_{t=0}=\bar{\omega} .
\end{array}\right.
$$

On pose alors $\bar{v}^{\varepsilon}(t, x)=\nabla^{\perp} \Delta^{-1} \bar{\omega}^{\varepsilon}(t, x), \check{v}^{\varepsilon}(t, x)=v^{\varepsilon}(t, x)-\bar{v}^{\varepsilon}(t, x)$. D'après [4] paragraphe 2.A, les fonctions $\check{v}^{\varepsilon}, \omega^{\prime \varepsilon}, \omega^{\prime \ell}$ vérifient les estimations (1.1.17), (1.1.22). En outre, d'après (1.2.44) et la positivité du noyau de la chaleur, on a $\omega^{\prime \varepsilon} \geq 0$ pour tout $\varepsilon$. Par contre, pour tous $(t, \varepsilon)$ fixés $x \rightarrow$ $\omega^{\varepsilon}(t, x)$ n'est plus à support compact mais seulement à décroissance rapide à l'infini. Cela suffit toutefois pour appliquer les raisonnements précédents qui entraînent que la limite d'une sous-suite de $\left(v^{\varepsilon}\right)_{\varepsilon}$ fournit une solution à l'équation d'Euler.

\section{NAPPES DE TOURBILLON SUR LES SURFACES COMPACTES}

2.1. Enoncé du théorème. Soit $(M, g)$ une surface riemannienne connexe, compacte, orientée. On notera $T M$ (resp. $T^{*} M$ ) le fibré tangent (resp. cotangent) à $M, \Lambda^{k} T M$ (resp. $\left.\Lambda^{k} T^{*} M\right) 0 \leq k \leq 2$ leurs puissances extérieures respectives. On désignera par $\mu$ la 2 -forme volume associée à la métrique et à l'orientation. On l'utilisera pour identifier distributions et courants sur $M$. Si $X$ est un champ de vecteurs sur $M$, on notera $L_{X}$ la dérivée de Lie dans la direction de $X$.

Si $F$ est un fibré sur $M$, on désignera par $L^{p}(M, F), C^{\infty}(M, F)$, $\mathscr{D}^{\prime}(M, F)$ l'espace des sections $L^{p}, C^{\infty}$, distributions de $F$ au dessus de $M$.

Si $(r, s)$ est un couple d'entiers positifs ou nuls, on notera $T_{s}^{r} M$ le fibré des champs de tenseurs de type $(r, s)$ sur $M$. La fibre de $T_{s}^{r} M$ en un point $x$ de $M$ est donc l'espace des applications multilinéaires sur $\left(T_{x}^{*} M \times \cdots \times T_{x}^{*} M\right) \times$ $\left(T_{x} M \times \cdots \times T_{x} M\right)$ à valeurs réelles, avec $r$ produits dans le premier facteur et $s$ dans le second. En particulier $T_{0}^{1} M=T M, T_{1}^{0} M=T^{*} M$. 
On désignera par la lettre $g$ non seulement la métrique sur $T M$ mais également celle qu'elle induit naturellement sur tous les fibrés $T_{s}^{r} M$.

On notera $\sim$ l'isomorphisme

$$
\begin{aligned}
T M & \rightarrow T^{*} M, \\
v & \rightarrow \tilde{v}
\end{aligned}
$$

associant au champ de vecteurs $v$ l'unique forme $\tilde{v}$ telle que pour tout champ de vecteurs $X$ sur $M, \tilde{v}(X)=g(v, X)$.

On notera $d_{k}: \Lambda^{k} T^{*} M \rightarrow \Lambda^{k+1} T^{*} M, k=0,1,2$, la différentielle extérieure sur $M, d_{k}^{*}: \Lambda^{k+1} T^{*} M \rightarrow \Lambda^{k} T^{*} M$ son adjoint pour la métrique et

$$
-\Delta_{k}=d_{k}^{*} d_{k}+d_{k-1} d_{k-1}^{*}
$$

l'opérateur de Laplace-Beltrami sur les $k$-formes.

Si $X$ est un champ de vecteurs sur $M$, on désigne par $\nabla_{X}$ la dérivation covariante le long de $X$ dans la connexion riemannienne. L'opérateur $\nabla_{X}$ agit sur $T_{s}^{r} M$ et si $t$ est une section $C^{\infty}$ de ce fibré, on a

$$
\begin{aligned}
\left(\nabla_{X} t\right) & \left(\omega_{1}, \ldots, \omega_{r}, X_{1}, \ldots, X_{s}\right) \\
= & X \cdot\left[t\left(\omega_{1}, \ldots, \omega_{r}, X_{1}, \ldots, X_{s}\right)\right] \\
& -\sum_{j=1}^{r} t\left(\omega_{1}, \ldots, \nabla_{X} \omega_{j}, \ldots, \omega_{r}, X_{1}, \ldots, X_{s}\right) \\
& -\sum_{j=1}^{s} t\left(\omega_{1}, \ldots, \omega_{r}, X_{1}, \ldots, \nabla_{X} X_{j}, \ldots, X_{s}\right)
\end{aligned}
$$

pour tous champs de vecteurs $C^{\infty} X_{1}, \ldots, X_{s}$ et tous champs de 1-formes $C^{\infty} \omega_{1}, \ldots, \omega_{r}$. En particulier, le fait que la dérivée covariante du tenseur métrique dans la connexion riemannienne soit nulle donne, d'après (2.1.3), la relation

$$
X \cdot g(Y, Z)=g\left(\nabla_{X} Y, Z\right)+g\left(Y, \nabla_{X} Z\right)
$$

valable pour tout triplet $(X, Y, Z)$ de champs de vecteurs $C^{\infty}$. En outre, (2.1.3) dans le cas où $t$ est une 1 -forme $\omega$ s'écrit

$$
\left(\nabla_{X} \omega\right)(Y)=X \cdot(\omega(Y))-\omega\left(\nabla_{X} Y\right)
$$

pour tout couple de champs de vecteurs $(X, Y)$. Cette dernière relation, combinée à (2.1.4), montre que

$$
\widetilde{\nabla_{X} Y}=\nabla_{X} \tilde{Y} .
$$

On note $\nabla: T_{s}^{r} M \rightarrow T_{s+1}^{r} M$ l'opérateur défini par

$$
(\nabla t)\left(\omega_{1}, \ldots, \omega_{r}, X_{0}, X_{1}, \ldots, X_{s}\right)=\left(\nabla_{X_{0}} t\right)\left(\omega_{1}, \ldots, \omega_{r}, X_{1}, \ldots, X_{s}\right)
$$


pour toutes familles $\left(\omega_{1}, \ldots, \omega_{r}\right)$ de 1 -formes $C^{\infty}$ et $\left(X_{0}, X_{1}, \ldots, X_{s}\right)$ de champs de vecteurs $C^{\infty}$. En particulier $\nabla$ opère de $T_{0}^{1} M=T M$ dans $T_{1}^{1} M$. On note

$$
\nabla^{*}: T_{1}^{1} M \rightarrow T_{0}^{1} M
$$

l'adjoint de $\nabla$ pour la métrique, i.e., l'opérateur défini pour toute section distribution $t$ de $T_{1}^{1} M$ et tout champ de vecteurs $X$ à coefficients $C^{\infty}$ par

$$
\int g\left(\nabla^{*} t, X\right) \mu=\int g(t, \nabla X) \mu .
$$

On désignera enfin par

$$
\tilde{\nabla}^{*}: T_{1}^{1} M \rightarrow T^{*} M=T_{1}^{0} M
$$

le composé de (2.1.8) et de l'isomorphisme (2.1.1).

Nous utiliserons le lemme suivant:

Lemme 2.1.1. Soit $v$ une section $C^{\infty}$ de $T M$ vérifiant $d_{0}^{*} \tilde{v}=0$. Alors

$$
\nabla_{v} \widetilde{v}=-\widetilde{\nabla}^{*}(v \otimes \tilde{v}) .
$$

Démonstration. On a si $X$ est un champ de vecteurs $C^{\infty}$ quelconque

$$
\begin{aligned}
\int \tilde{\nabla}^{*}(v \otimes \tilde{v})(X) \mu & =\int g(v \otimes \tilde{v}, \nabla X) \mu=\int \nabla X(\tilde{v} \otimes v) \mu \\
& =\int\left(\nabla_{v} X\right)(\tilde{v}) \mu=\int g\left(\nabla_{v} X, v\right) \mu \\
& =\int d_{0}(g(X, v))(v) \mu-\int g\left(X, \nabla_{v} v\right) \mu \\
& =\int g(X, v) d_{0}^{*} \tilde{v} \mu-\int\left(\nabla_{v} \tilde{v}\right)(X) \mu
\end{aligned}
$$

où l'avant-dernière égalité résulte de (2.1.4) et la dernière de (2.1.6). Comme $d_{0}^{*} \tilde{v}=0$, on a bien (2.1.11).

Si $U$ est un ouvert de $M$ sur lequel on dispose d'un système de coordonnées locales $x=\left(x^{1}, x^{2}\right)$ compatibles avec l'orientation, on note $\left(g_{i j}\right)_{1 \leq i, j \leq 2}$ la matrice de $g$ et $\left(g^{i j}\right)_{1 \leq i, j \leq 2}$ la matrice inverse. On utilisera les conventions de sommation d'Einstein.

L'isomorphisme (2.1.1) associe alors au champ de vecteurs $v=v^{i} \partial / \partial x^{i}$ la 1 -forme

$$
\tilde{v}=\tilde{v}_{j} d x^{j}=g_{i j} v^{i} d x^{j} .
$$

La forme volume $\mu$ vaut $(\operatorname{det} g)^{1 / 2} d x^{1} \wedge d x^{2}$ et on a

$$
d_{0}^{*} \tilde{v}=-(\operatorname{det} g)^{-1 / 2} \frac{\partial}{\partial x^{i}}\left[(\operatorname{det} g)^{1 / 2} v^{i}\right] .
$$

Si $f$ est une fonction sur $M$, on a

$$
\Delta_{0} f=(\operatorname{det} g)^{-1 / 2} \frac{\partial}{\partial x^{j}}\left[(\operatorname{det} g)^{1 / 2} g^{i j} \frac{\partial f}{\partial x^{i}}\right] .
$$


Le symbole principal de $\Delta_{0}$ est donc $-g^{i j}(x) \xi_{i} \xi_{j}$. Si $\omega$ est une 2-forme et si on écrit $\omega=f \mu$ avec $f$ fonction sur $M$, alors $\Delta_{2} \omega=\left(\Delta_{0} f\right) \mu$ où $\Delta_{0} f$ est donné par (2.1.14).

L'opérateur (2.1.10) associe au tenseur de type $(1,1) \quad t=t_{\beta}^{\alpha} \partial / \partial x^{\alpha} \otimes d x^{\beta}$ la 1-forme différentielle

$$
\left[-(\operatorname{det} g)^{-1 / 2} \frac{\partial}{\partial x^{j}}\left(t_{\beta}^{\alpha} g_{\alpha i} g^{\beta j}(\operatorname{det} g)^{1 / 2}\right)+\Gamma_{i j}^{k} t_{\beta}^{\alpha} g_{\alpha k} g^{\beta j}\right] d x^{i},
$$

où les $\Gamma_{i j}^{k}$ sont les symboles de Christoffel.

Enfin, introduisons une dernière notation: si $\omega \in L^{p}\left(M, \Lambda^{2} T^{*} M\right) \quad(1 \leq p \leq$ $+\infty)$, on a $\omega=f \mu$ avec $f \in L^{p}(M)$ et on notera $\|\omega\|_{L^{p}}=\left(\int_{M}|f|^{p} \mu\right)^{1 / p}$.

Décomposition de Hodge. Les opérateurs

$$
\begin{aligned}
& -\Delta_{0}: C^{\infty}(M) \rightarrow C^{\infty}(M), \\
& -\Delta_{0}: \mathscr{D}^{\prime}(M) \rightarrow \mathscr{D}^{\prime}(M)
\end{aligned}
$$

ont pour noyau l'espace des fonctions constantes. L'image (fermée) du second est l'orthogonal du noyau du premier, i.e., l'espace

$$
\mathscr{D}_{0}^{\prime}(M)=\left\{f \in \mathscr{D}^{\prime}(M) ; \int f \mu=0\right\}
$$

et $-\Delta_{0}$ est un isomorphisme de $\mathscr{D}_{0}^{\prime}(M)$ sur lui-même. On note

$$
K_{0}: \mathscr{D}_{0}^{\prime}(M) \rightarrow \mathscr{D}_{0}^{\prime}(M)
$$

son inverse. De même si $\mathscr{D}_{0}^{\prime}\left(M, \Lambda^{2} T^{*} M\right)$ désigne l'espace des sections distributions $\omega$ de $\Lambda^{2} T^{*} M$ au-dessus de $M$ vérifiant $\int \omega=0$, on note

$$
K_{1}: \mathscr{D}_{0}^{\prime}\left(M, \Lambda^{2} T^{*} M\right) \rightarrow \mathscr{D}_{0}^{\prime}\left(M, \Lambda^{2} T^{*} M\right)
$$

l'opérateur défini par $K_{1}(f \mu)=\left(K_{0} f\right) \mu$ pour $f \in \mathscr{D}_{0}^{\prime}(M)$. L'opérateur $K_{1}$ est l'inverse de $-\Delta_{2}$.

Si $\alpha$ est une section distribution de $T^{*} M$, on voit donc que:

$$
h=\alpha-d_{0} K_{0} d_{0}^{*} \alpha-d_{1}^{*} K_{1} d_{1} \alpha
$$

vérifie $d_{1} h=0, d_{0}^{*} h=0$, i.e., est une forme harmonique (en particulier, $h$ est $\left.C^{\infty}\right)$. Autrement dit, l'unique décomposition de Hodge de la forme $\alpha$ s'écrit:

$$
\alpha=d_{0} K_{0} d_{0}^{*} \alpha+d_{1}^{*} K_{1} d_{1} \alpha+H(\alpha),
$$

où $H$ désigne le projecteur naturel sur l'espace (de dimension finie) des 1formes harmoniques sur $M$. Si l'on choisit une base $\left(\alpha_{1}, \ldots, \alpha_{d}\right)$ de cet espace, orthonormale pour le produit scalaire provenant de la métrique, on a

$$
H(\alpha)=\sum_{k=1}^{d}\left(\int g\left(\alpha, \alpha_{k}\right) \mu\right) \alpha_{k} .
$$

Nous allons maintenant énoncer le résultat d'existence pour les nappes de tourbillon sur une variété compacte. 
Théorème 2.1.2. Soit $v_{0}$ un élément de $L^{2}(M, T M)$ vérifiant $d_{0}^{*} \tilde{v}_{0}=0$. Supposons que le tourbillon $\omega_{0}=d_{1} \tilde{v}_{0}$ de $v_{0}$ s'écrit $\omega_{0}=\omega_{0}^{\prime}+\omega_{0}^{\prime \prime}$ avec $\omega_{0}^{\prime}=a_{0} \mu$ où $a_{0}$ est une mesure positive ou nulle sur $M$ et avec $\omega_{0}^{\prime \prime} \in L^{p}\left(M, \Lambda^{2} T^{*} M\right)$ pour un réel $p \in] 1,+\infty]$.

Il existe alors $v \in L^{\infty}\left(\mathbb{R}, L^{2}(M, T M)\right)$ et $p \in L^{\infty}\left(\mathbb{R}, \mathscr{D}^{\prime}(M)\right)$ solutions $d u$ système d'Euler sur $M$

$$
\left\{\begin{array}{l}
\partial \tilde{v} / \partial t-\tilde{\nabla}^{*}(v \otimes \tilde{v})=-d_{0} p \\
d_{0}^{*} \tilde{v}=0 \\
\left.\tilde{v}\right|_{t=0}=\tilde{v}_{0} .
\end{array}\right.
$$

De plus on a, pour tout $t \in \mathbb{R}$ l'inégalité:

$$
\int g(v(t, \cdot), v(t, \cdot)) \mu \leq \int g\left(v_{0}, v_{0}\right) \mu .
$$

En outre, si pour tout $t \in \mathbb{R}$ on pose $\omega(t, \cdot)=d_{1} \tilde{v}(t, \cdot)$, on a une décomposition $\omega(t, \cdot)=\omega_{t}^{\prime}+\omega_{t}^{\prime \prime}$ où $\omega_{t}^{\prime}=a_{t}^{\prime} \mu$ avec $a_{t}^{\prime}$ mesure positive ou nulle telle que $\int \omega_{t}^{\prime}=\int \omega_{0}^{\prime}$ et où $\omega_{t}^{\prime \prime} \in L^{p}\left(M, \Lambda^{2} T^{*} M\right)$ et vérifie

$$
\left\|\omega_{t}^{\prime \prime}\right\|_{L^{p}(M)} \leq\left\|\omega_{0}^{\prime \prime}\right\|_{L^{p}(M)} .
$$

Remarques.

-D'après l'injection de Sobolev $L^{p}(M) \hookrightarrow H^{-1}(M)$ pour $p>1, \omega_{0}^{\prime \prime}$ et donc aussi $\omega_{0}^{\prime}$ sont dans $H^{-1}(M)$.

-Dans le cas où la donnée (et donc la solution $v$ ) est régulière, il résulte du Lemme 2.1.1 que la première équation (2.1.22) s'écrit

$$
\frac{\partial \tilde{v}}{\partial t}+\nabla_{v} \tilde{v}=-d_{0} p
$$

qui est bien l'expression usuelle de l'équation d'Euler sur une variété compacte (cf. $[1, \S 8.2])$.

-L'énoncé précédent contient le cas de l'équation d'Euler sur le plan à données nappes de tourbillon $\mathbb{Z}^{2}$-périodiques: il suffit en effet de prendre pour variété $M$ le tore muni de la métrique plate.

Comme dans le cas du plan, la preuve du théorème repose sur l'équation équivalente à (2.1.22) obtenue en éliminant la pression. Pour cela, nous aurons besoin du lemme suivant:

Lemme 2.1.3. Soit $\delta$ le tenseur de type $(1,1)$ donné dans un système quelconque de coordonnées locales par

$$
\delta=\delta_{j}^{i} \partial / \partial x^{i} \otimes d x^{j}
$$

$\left(\delta_{j}^{i}=\right.$ symbole de Kronecker). Alors si $f \in \mathscr{D}^{\prime}(M)$, on a

$$
-\tilde{\nabla}^{*}(f \delta)=d_{0} f .
$$


Démonstration. Dans un système de coordonnées locales, on a, d'après (2.1.15)

$$
\begin{aligned}
-\tilde{\nabla}^{*}(f \delta) & =\left\{(\operatorname{det} g)^{-1 / 2} \frac{\partial}{\partial x^{j}}\left[(\operatorname{det} g)^{1 / 2} \delta_{i}^{j} f\right]-\Gamma_{i j}^{k} \delta_{k}^{j} f\right\} d x^{i} \\
& =\frac{\partial f}{\partial x^{i}} d x^{i}-\left\{-\frac{1}{2}(\operatorname{det} g)^{-1} \frac{\partial}{\partial x^{i}}(\operatorname{det} g)+\sum_{j} \Gamma_{i j}^{j}\right\} f d x^{i} .
\end{aligned}
$$

Or

$$
\Gamma_{i j}^{k}=\frac{1}{2} g^{k s}\left(\frac{\partial g_{s i}}{\partial x^{j}}-\frac{\partial g_{i j}}{\partial x^{s}}+\frac{\partial g_{j s}}{\partial x^{i}}\right), \quad \text { d'où } \sum_{j} \Gamma_{i j}^{j}=\frac{1}{2} \sum_{j, s} g^{j s} \frac{\partial g_{j s}}{\partial x^{i}} .
$$

Utilisant les relations

$$
g^{12}=-(\operatorname{det} g)^{-1} g_{12}, \quad g^{11}=(\operatorname{det} g)^{-1} g_{22}, \quad g^{22}=(\operatorname{det} g)^{-1} g_{11}
$$

on constate que cette expression n'est autre que $\frac{1}{2}(\operatorname{det} g)^{-1} \frac{\partial}{\partial x^{i}}(\operatorname{det} g)$. Le lemme est prouvé.

Nous allons en déduire

Proposition 2.1.4. Soit $v_{0} \in L^{2}(M, T M)$ vérifiant $d_{0}^{*} \tilde{v}_{0}=0$. Les conditions suivantes sont équivalentes:

(i) Il existe $v \in L^{\infty}\left(\mathbb{R}, L^{2}(M, T M)\right)$ et $p \in L^{\infty}\left(\mathbb{R}, \mathscr{D}^{\prime}(M)\right)$ solutions de (2.1.22).

(ii) Il existe $v \in L^{\infty}\left(\mathbb{R}, L^{2}(M, T M)\right)$ solution de

$$
\left\{\begin{array}{l}
\frac{\partial \tilde{v}}{\partial t}-\left[d_{1}^{*} K_{1} d_{1}+H\right]\left(\tilde{\nabla}^{*}\left(v \otimes \tilde{v}-\frac{1}{2} g(v, v) \delta\right)\right)=0 \\
\left.\tilde{v}\right|_{t=0}=\tilde{v}_{0}
\end{array}\right.
$$

Démonstration. Supposons (i) vérifié. Alors la condition $d_{0}^{*} \tilde{v}=0$ entraîne que dans la décomposition de Hodge (2.1.20) de $\partial \tilde{v} / \partial t$ le premier terme est nul. L'unicité de la décomposition de Hodge des deux membres de la première équation (2.1.22) entraîne alors que $v$ vérifie

$$
\partial \tilde{v} / \partial t-\left[d_{1}^{*} K_{1} d_{1}+H\right]\left(\tilde{\nabla}^{*}(v \otimes \tilde{v})\right)=0
$$

D'après le Lemme 2.1.3 et l'expression (2.1.21) de $H$

$$
\begin{aligned}
d_{1} \tilde{\nabla}^{*}(v \otimes \tilde{v}) & =d_{1} \tilde{\nabla}^{*}\left(v \otimes \tilde{v}-\frac{1}{2} g(v, v) \delta\right), \\
H\left(\tilde{\nabla}^{*}(v \otimes \tilde{v})\right) & =H\left(\tilde{\nabla}^{*}\left(v \otimes \tilde{v}-\frac{1}{2} g(v, v) \delta\right)\right),
\end{aligned}
$$

donc (ii) est vrai.

Réciproquement, si (ii) est réalisé, alors $(\partial / \partial t) d_{0}^{*} \tilde{v}=0$ d'où $d_{0}^{*} \tilde{v}=0$. En outre $v$ vérifie $(2.1 .31)$ et le membre de gauche de cette équation ne differe du membre de gauche de la première équation (2.1.22) que par une 1-forme exacte. La proposition est démontrée.

Comme dans la première partie, nous allons maintenant montrer que la proposition précédente réduit la preuve du Théorème 2.1.2 à un résultat de 
passage à la limite sur les coefficients du tenseur $v^{\varepsilon} \otimes \tilde{v}^{\varepsilon}-\frac{1}{2} g\left(v^{\varepsilon}, v^{\varepsilon}\right) \delta$ lorsque $v^{\varepsilon}$ est une suite de solutions de l'équation d'Euler à données régularisées.

Pour $\varepsilon \in] 0,1]$, posons

$$
\begin{aligned}
\tilde{v}_{0}^{\varepsilon} & =e^{\varepsilon \Delta_{1}} \tilde{v}_{0}, & \omega_{0}^{\varepsilon}=e^{\varepsilon \Delta_{2}} \omega_{0}, \\
\omega_{0}^{\prime \varepsilon} & =e^{\varepsilon \Delta_{2}} \omega_{0}^{\prime}, & \omega_{0}^{\prime \prime \varepsilon}=e^{\varepsilon \Delta_{2}} \omega_{0}^{\prime \prime} .
\end{aligned}
$$

Comme $d_{1} \Delta_{1}=\Delta_{2} d_{1}$ on a $\omega_{0}^{\varepsilon}=d_{1} \tilde{v}_{0}^{\varepsilon}$. On note $v_{0}^{\varepsilon}$ l'image réciproque de $\tilde{v}_{0}^{\varepsilon}$ par (2.1.1). Pour tout $\varepsilon \in] 0,1], v_{0}^{\varepsilon} \in C^{\infty}(M, T M)$ et $\omega_{0}^{\varepsilon}, \omega_{0}^{\ell}, \omega_{0}^{\prime \prime}$ sont dans $C^{\infty}\left(M, \Lambda^{2} T^{*} M\right)$. La positivité du noyau de la chaleur entraîne que $\omega_{0}^{\prime \varepsilon}=a_{0}^{\prime \varepsilon} \mu$ avec $a_{0}^{\prime \varepsilon}=e^{\varepsilon \Delta_{0}} a_{0}^{\prime}$, fonction positive ou nulle sur $M$. On a en outre

$$
\begin{gathered}
\int g\left(v_{0}^{\varepsilon}, v_{0}^{\varepsilon}\right) \mu \leq \int g\left(v_{0}, v_{0}\right) \mu, \\
\left\|\omega_{0}^{\prime \varepsilon}\right\|_{L^{p}} \leq\left\|\omega_{0}^{\prime \prime}\right\|_{L^{p}} \\
\int \omega_{0}^{\prime \varepsilon}=\int \omega_{0}^{\prime} .
\end{gathered}
$$

Enfin si $\varepsilon \rightarrow 0+, v_{0}^{\varepsilon}$ (resp. $\omega_{0}^{\prime \varepsilon}$, resp. $\left.\omega_{0}^{\prime \prime}\right)$ converge au sens des distributions vers $v_{0}\left(\right.$ resp. $\omega_{0}^{\prime}$, resp. $\left.\omega_{0}^{\prime \prime}\right)$.

Pour tout $\varepsilon>0$, il existe d'après [6, Théorème 15.2; 9] une solution globale $v^{\varepsilon} \in C^{\infty}\left(\mathbb{R}, C^{\infty}(M, T M)\right), p^{\varepsilon} \in C^{\infty}\left(\mathbb{R}, C^{\infty}(M)\right)$ à l'équation

$$
\left\{\begin{array}{l}
\partial \tilde{v}^{\varepsilon} / \partial t+\nabla_{v^{\varepsilon}} \tilde{v}^{\varepsilon}=-d p^{\varepsilon}, \\
d_{0}^{*} \tilde{v}^{\varepsilon}=0, \\
\left.\tilde{v}^{\varepsilon}\right|_{t=0}=\tilde{v}_{0}^{\varepsilon} .
\end{array}\right.
$$

Comme, utilisant (2.1.4), on voit que

$$
\left(\partial / \partial t+v^{\varepsilon}\right)\left(g\left(v^{\varepsilon}(t, \cdot), v^{\varepsilon}(t, \cdot)\right)\right)=-2 g\left(d p, \tilde{v}^{\varepsilon}(t, \cdot)\right)
$$

et comme $d_{0}^{*} \tilde{v}^{\varepsilon}=0$, on a pour tout $t \in \mathbb{R}$

$$
\int g\left(v^{\varepsilon}(t, \cdot), v^{\varepsilon}(t, \cdot)\right) \mu=\int g\left(v_{0}^{\varepsilon}, v_{0}^{\varepsilon}\right) \mu .
$$

D'autre part, le tourbillon $\omega^{\varepsilon}=d \tilde{v}^{\varepsilon}$ vérifie d'après [1, Proposition 8.2.6], l'équation

$$
\left\{\begin{array}{l}
\partial \omega^{\varepsilon} / \partial t+L_{v^{\varepsilon}} \omega^{\varepsilon}=0 \\
\left.\omega^{\varepsilon}\right|_{t=0}=\omega_{0}^{\varepsilon}
\end{array}\right.
$$

ce qui, si l'on note $\varphi_{t}^{\varepsilon}$ le flot du champ de vecteurs $v^{\varepsilon}$ à l'instant $t$, s'écrit encore

$$
\varphi_{t}^{\varepsilon *} \omega^{\varepsilon}(t, \cdot)=\omega_{0}^{\varepsilon}
$$

On décompose $\omega^{\varepsilon}=\omega^{1 \varepsilon}+\omega^{\prime \prime \varepsilon}$ où $\omega^{\prime \varepsilon}$ et $\omega^{\prime \prime \varepsilon}$ sont définis par les relations

$$
\varphi_{t}^{\varepsilon *} \omega^{\prime \varepsilon}(t, \cdot)=\omega_{0}^{\ell \varepsilon}, \quad \varphi_{t}^{\varepsilon *} \omega^{\prime \prime \varepsilon}(t, \cdot)=\omega_{0}^{\prime \varepsilon} .
$$

Alors $\omega^{\ell}$ est positive ou nulle et comme le jacobien de $\varphi_{t}^{\varepsilon}$ est identiquement égal à 1 , on a

$$
\begin{aligned}
& \left\|\omega^{\prime \varepsilon}(t, \cdot)\right\|_{L^{1}}=\left\|\omega_{0}^{\prime \varepsilon}\right\|_{L^{1}}, \\
& \left\|\omega^{\prime \prime \varepsilon}(t, \cdot)\right\|_{L^{p}}=\left\|\omega_{0}^{\prime \prime \varepsilon}\right\|_{L^{p}} \text {. }
\end{aligned}
$$


On déduit donc de (2.1.34), (2.1.37), et (2.1.41) les estimations uniformes suivantes

$$
\begin{gathered}
\left.\left.\left\|v^{\varepsilon}\right\|_{L^{\infty}\left(\mathbb{R}, L^{2}(M, T M)\right)} \leq\left\|v_{0}\right\|_{L^{2}(M, T M)} \quad \forall \varepsilon \in\right] 0,1\right], \\
\left.\left.\int \omega^{i \varepsilon}(t, \cdot)=\int \omega_{0}^{\prime} \quad \forall t \in \mathbb{R}, \forall \varepsilon \in\right] 0,1\right], \\
\left.\left.\left\|\omega^{\prime \prime \varepsilon}\right\|_{L^{\infty}\left(\mathbb{R}, L^{p}\left(M, \Lambda^{2} T^{*} M\right)\right)} \leq\left\|\omega_{0}^{\prime \prime}\right\|_{L^{p}\left(M, \Lambda^{2} T^{*} M\right)} \quad \forall \varepsilon \in\right] 0,1\right] .
\end{gathered}
$$

Enfin, puisque d'après la Proposition 2.1.4, $v^{\varepsilon}$ vérifie la première équation (2.1.30), $\left(v^{\varepsilon}\right)_{\varepsilon}$ est borné dans l'espace $\operatorname{Lip}\left(\mathbb{R}, \mathscr{D}^{\prime}(M, T M)\right)$ des fonctions lipschitziennes de $t$ à valeurs dans les sections distributions de $T M$. Par conséquent $\left(\omega^{\varepsilon}\right)_{\varepsilon}$ est bornée dans $\operatorname{Lip}\left(\mathbb{R}, \mathscr{D}^{\prime}\left(M, \Lambda^{2} T^{*} M\right)\right)$.

D'après les inégalités (2.1.42), on peut extraire des suites $\left(v^{\varepsilon}\right)_{\varepsilon \in] 0,1]}$, $\left(\omega^{\varepsilon}\right)_{\varepsilon \in] 0,1]},\left(\omega^{\prime \varepsilon}\right)_{\varepsilon \in] 0,1]},\left(\omega^{\prime \ell}\right)_{\varepsilon \in] 0,1]}$ des sous-suites $\left(v^{\varepsilon}\right)_{\varepsilon \in J},\left(\omega^{\varepsilon}\right)_{\varepsilon \in J},\left(\omega^{\prime \varepsilon}\right)_{\varepsilon \in J}$, $\left(\omega^{\prime \prime \varepsilon}\right)_{\varepsilon \in J}$ où $\left.\left.J \subset\right] 0,1\right]$ avec $0 \in \bar{J}$, convergeant au sens des distributions vers des limites $v \in L^{\infty}\left(\mathbb{R}, L^{2}(M, T M)\right), \omega \in L^{\infty}\left(\mathbb{R}, H^{-1}\left(M, \Lambda^{2} T^{*} M\right)\right), \omega^{\prime}$ fonction $L^{\infty}$ de $t$ à valeurs dans les sections mesures positives ou nulles de $\Lambda^{2} T^{*} M$ et $\omega^{\prime \prime} \in L^{\infty}\left(\mathbb{R}, L^{p}\left(M, \Lambda^{2} T^{*} M\right)\right)$. Pour prouver le théorème, il nous suffit donc d'après la Proposition 2.1.4 de montrer:

Proposition 2.1.5. Si $\varepsilon$ tend vers 0 dans $J, v^{\varepsilon} \otimes \tilde{v}^{\varepsilon}-\frac{1}{2} g\left(v^{\varepsilon}, v^{\varepsilon}\right) \delta$ converge au sens des distributions sur $\mathbb{R} \times M$ vers $v \otimes \tilde{v}-\frac{1}{2} g(v, v) \delta$.

La preuve de cette proposition occupe le paragraphe suivant.

2.2. Passage à la limite. Il nous suffit de prouver la Proposition 2.1.5 localement ce qui permet de se restreindre à un domaine de carte muni de coordonnées locales $\left(x^{1}, x^{2}\right)$ centrées en 0 . On a alors, en notant $\partial_{i}=\partial / \partial x^{i}, i=1,2$,

$$
v^{\varepsilon}=v^{i, \varepsilon} \partial_{i}, \quad \tilde{v}^{\varepsilon}=\tilde{v}_{j}^{\varepsilon} d x^{j}, \quad \tilde{v}_{j}^{\varepsilon}=g_{i j} v^{i, \varepsilon}
$$

et on doit prouver

Théorème 2.2.1. Soit $\left(v^{\varepsilon}\right)_{\varepsilon \in J}$ une suite de $L^{\infty}\left(\mathbb{R}, C^{\infty}(M, T M)\right)$ bornée dans $L^{\infty}\left(\mathbb{R}, L^{2}(M, T M)\right)$ et dans $\operatorname{Lip}\left(\mathbb{R}, \mathscr{D}^{\prime}(M, T M)\right)$ telle que $d_{0}^{*} \tilde{v}^{\varepsilon}=$ 0 et que $\omega^{\varepsilon}=d_{1} \tilde{v}^{\varepsilon}$ s'écrive $\omega^{\varepsilon}=\omega^{\varepsilon}+\omega^{\prime \prime}$ où $\omega^{\prime \varepsilon}$ (resp. $\left.\omega^{\prime \prime \varepsilon}\right)$ est dans $L^{\infty}\left(\mathbb{R}, C^{\infty}\left(M, \Lambda^{2} T^{*} M\right)\right)$, bornée dans $L^{\infty}\left(\mathbb{R}, L^{1}\left(M, \Lambda^{2} T^{*} M\right)\right)$ (resp. dans $L^{\infty}\left(\mathbb{R}, L^{p}\left(M, \Lambda^{2} T^{*} M\right)\right)$ pour un certain réel $\left.p>1\right)$ et où $\omega^{\prime} \geq 0$. Supposons que lorsque $\varepsilon$ tend vers 0 dans $J, v^{\varepsilon}, \omega^{\varepsilon}, \omega^{\varepsilon}, \omega^{\prime \prime}$ convergent au sens des distributions vers des limites $v, \omega, \omega^{\prime}, \omega^{\prime \prime}$. Alors si $\varphi \in C_{0}^{\infty}\left(\mathbb{R}^{2}\right)$ est à support dans l'image du domaine de carte considéré et $\psi \in C_{0}^{\infty}(\mathbb{R})$ on a

$$
\begin{aligned}
& \int v^{1, \varepsilon}(t, z) \tilde{v}_{2}^{\varepsilon}(t, z) \varphi(z) \psi(t) d z d t \\
& \quad \rightarrow \int v^{1}(t, z) \tilde{v}_{2}(t, z) \varphi(z) \psi(t) d z d t
\end{aligned}
$$




$$
\begin{gathered}
\int v^{2, \varepsilon}(t, z) \tilde{v}_{1}^{\varepsilon}(t, z) \varphi(z) \psi(t) d z d t \\
\rightarrow \int v^{2}(t, z) \tilde{v}_{1}(t, z) \varphi(z) \psi(t) d z d t \\
\int\left(v^{2, \varepsilon}(t, z) \tilde{v}_{2}^{\varepsilon}(t, z)-v^{1, \varepsilon}(t, z) \tilde{v}_{1}^{\varepsilon}(t, z)\right) \varphi(z) \psi(t) d z d t \\
\rightarrow \int\left(v^{2}(t, z) \tilde{v}_{2}(t, z)-v^{1}(t, z) \tilde{v}_{1}(t, z)\right) \varphi(z) \psi(t) d z d t
\end{gathered}
$$

lorsque $\varepsilon$ tend vers 0 dans $J$.

La méthode utilisée est la même que dans le cas de l'équation d'Euler sur $\mathbb{R}^{2}$. Il s'agit d'abord d'exprimer les composantes de la vitesse $v^{1, \varepsilon}, v^{2, \varepsilon}$ en fonction de $\omega^{\varepsilon}$. On peut écrire $\omega^{\varepsilon}(x)=a^{\varepsilon}(x) d x^{1} \wedge d x^{2}$. On a alors $a^{\varepsilon}(x)=$ $\partial_{1} \tilde{v}_{2}^{\varepsilon}-\partial_{2} \tilde{v}_{1}^{\varepsilon}$.

Soient $\theta$ et $\bar{\theta}$ deux fonctions $C^{\infty}$ à support compact assez voisin de $\operatorname{Supp} \varphi$, positives ou nulles, vérifiant $\theta \equiv 1$ au voisinage de $\operatorname{Supp} \varphi$ et $\bar{\theta} \equiv 1$ au voisinage de $\operatorname{Supp} \theta$.

Lemme 2.2.2. Il existe un opérateur pseudo-différentiel $G$ (resp. $R^{1}$, resp. $R^{2}$ ) opérant de l'espace des distributions sur $\mathbb{R}^{2}$ à valeurs scalaires (resp. à valeurs dans $\mathbb{R}^{2}$ ) dans l'espace des distributions scalaires sur $\mathbb{R}^{2}$, d'ordre -2 (resp. d'ordre -1) tel que l'on ait au voisinage de $\operatorname{Supp} \varphi$

$$
\left\{\begin{array}{l}
v^{1, \varepsilon}=-G \partial_{2}\left(\theta a^{\varepsilon}\right)+R^{1}\left(\bar{\theta} v^{\varepsilon}\right), \\
v^{2, \varepsilon}=G \partial_{1}\left(\theta a^{\varepsilon}\right)+R^{2}\left(\bar{\theta} v^{\varepsilon}\right) .
\end{array}\right.
$$

On peut en outre supposer que la symbole complet de $G$ est égal à

$$
\sigma(G)(x, \xi)=-(\operatorname{det} g(x))^{-1}\left(g^{i j} \xi_{i} \xi_{j}\right)^{-1} \gamma(\xi),
$$

où $\gamma \in C^{\infty}\left(\mathbb{R}^{2}\right)$ est paire et vérifie $\gamma \equiv 1$ pour $|\xi| \geq 1, \gamma \equiv 0$ pour $|\xi| \leq \frac{1}{2}$.

Démonstration. D'après $(2.1 .13)$ la relation $d_{0}^{*} \tilde{v}^{\varepsilon}=0$ entraîne que $\partial_{1} v^{1, \varepsilon}+$ $\partial_{2} v^{2, \varepsilon}$ est combinaison linéaire à coefficients $C^{\infty}$ de $v^{1, \varepsilon}, v^{2, \varepsilon}$. On a donc

$$
\begin{aligned}
\partial_{1}\left(\theta a^{\varepsilon}\right) & =\theta \partial_{1}^{2} \tilde{v}_{2}^{\varepsilon}-\theta \partial_{1} \partial_{2} \tilde{v}_{1}^{\varepsilon}+\left[\partial_{1}, \theta\right] a^{\varepsilon} \\
& =\theta\left(g_{2 j} \partial_{1}^{2} v^{j, \varepsilon}-g_{1 j} \partial_{1} \partial_{2} v^{j, \varepsilon}\right)+Q_{1}\left(v^{\varepsilon}\right) \\
& =\theta\left(-2 g_{21} \partial_{1} \partial_{2} v^{2, \varepsilon}+g_{22} \partial_{1}^{2} v^{2, \varepsilon}+g_{11} \partial_{2}^{2} v^{2, \varepsilon}\right)+Q_{2}\left(v^{\varepsilon}\right),
\end{aligned}
$$

où $Q_{1}$ et $Q_{2}$ sont des opérateurs différentiels de degré 1 opérant de $\mathbb{R}^{2}$ dans $\mathbb{R}$.

D’après les relations (2.1.29), $\partial_{1}\left(\theta a^{\varepsilon}\right)$ est donc égal à

$$
(\operatorname{det} g)\left[g^{i j} \partial_{i} \partial_{j}\right]\left(\theta v^{2, \varepsilon}\right)+Q\left(v^{\varepsilon}\right)
$$

où $Q$ est un nouvel opérateur différentiel de degré 1. La seconde égalité (2.2.3) en résulte. La première se prouve de même. 
Comme $\left(v^{\varepsilon}\right)_{\varepsilon \in J}$ est bornée dans

$$
\operatorname{Lip}\left(\mathbb{R}, \mathscr{D}^{\prime}(M, T M)\right) \cap L^{\infty}\left(\mathbb{R}, L^{2}(M, T M)\right),
$$

pour tout $t \in \mathbb{R}, v^{\varepsilon}(t, \cdot)$ converge faiblement dans $L^{2}$ vers $v(t, \cdot)$ (cf. Lemme 1.2.1). Puisque $R^{j}, j=1,2$, est d'ordre $-1, R^{j}\left(\bar{\theta} v^{\varepsilon}(t, \cdot)\right)$ converge fortement dans $L^{2}$ vers $R^{j}(\bar{\theta} v(t, \cdot))$ et $R^{j}\left(\bar{\theta} v^{\varepsilon}\right)$ est borné dans $L^{\infty}\left(\mathbb{R}, L^{2}(M)\right)$. D'autre part, $\left(\theta a^{\varepsilon}\right)_{\varepsilon \in J}$ est bornée dans $\operatorname{Lip}\left(\mathbb{R}, \mathscr{D}^{\prime}(M)\right) \cap L^{\infty}\left(\mathbb{R}, H^{-1}(M)\right)$. Il en résulte que pour tout $t \in \mathbb{R}, G \partial_{j}\left(\theta a^{\varepsilon}(t, \cdot)\right)$ converge faiblement dans $L^{2}$ vers $G \partial_{j}(\theta a(t, \cdot))$. De plus, $G \partial_{j}\left(\theta a^{\varepsilon}\right)$ est bornée dans $L^{\infty}\left(\mathbb{R}, L^{2}(M)\right)$. Utilisant alors, pour tout $t \in \mathbb{R}$, le fait que le produit d'une suite faiblement convergente par une suite fortement convergente converge au sens des distributions, puis le théorème de convergence dominée (pour traiter l'intégrale en $t$ ), on déduit de (2.2.3):

$$
\begin{aligned}
& \lim _{\substack{\varepsilon \in 0 \\
\varepsilon \in J}} \int\left(v^{1, \varepsilon} \tilde{v}_{2}^{\varepsilon}-v^{1} \tilde{v}_{2}\right)(t, z) \varphi(z) \psi(t) d z d t \\
& =\lim _{\substack{\varepsilon \rightarrow 0 \\
\varepsilon \in J}} \int\left[G \partial_{2}\left(\theta a^{\varepsilon}\right)\left(g_{12} G \partial_{2}\left(\theta a^{\varepsilon}\right)-g_{22} G \partial_{1}\left(\theta a^{\varepsilon}\right)\right)\right. \\
& \left.\quad+G \partial_{2}(\theta a)\left(-g_{12} G \partial_{2}(\theta a)+g_{22} G \partial_{1}(\theta a)\right)\right] \varphi(z) \psi(t) d z d t
\end{aligned}
$$

et, pour prouver $(2.2 .1)_{1}$, il nous suffit de voir que cette dernière expression est nulle. Faisant le même raisonnement pour $v^{2, \varepsilon} \tilde{v}_{1}^{\varepsilon}$ et $v^{2, \varepsilon} \tilde{v}_{2, \varepsilon}-v^{1, \varepsilon} \tilde{v}_{1, \varepsilon}$, on voit que le Théorème 2.2.1 est corollaire de la proposition suivante:

Proposition 2.2.3. Avec les notations précédentes, on a

$$
\begin{array}{r}
\lim _{\substack{\varepsilon \rightarrow 0 \\
\varepsilon \in J}} \int G \partial_{k}\left(\theta a^{\varepsilon}\right)\left[\left(-g_{1 k} G \partial_{2}+g_{2 k} G \partial_{1}\right)\left(\theta a^{\varepsilon}\right)\right](t, z) \varphi(z) \psi(t) d z d t \\
\quad=\int G \partial_{k}(\theta a)\left[\left(-g_{1 k} G \partial_{2}+g_{2 k} G \partial_{1}\right)(\theta a)\right](t, z) \varphi(z) \psi(t) d z d t
\end{array}
$$

pour $k=1,2$ et

$$
\begin{aligned}
\lim _{\substack{\varepsilon \in J \\
\varepsilon \in J}} \int\{ & G \partial_{1}\left(\theta a^{\varepsilon}\right)\left[\left(-g_{12} G \partial_{2}+g_{22} G \partial_{1}\right)\left(\theta a^{\varepsilon}\right)\right](t, z) \\
& \left.+G \partial_{2}\left(\theta, a^{\varepsilon}\right)\left[\left(-g_{11} G \partial_{2}+g_{12} G \partial_{1}\right)\left(\theta a^{\varepsilon}\right)\right](t, z)\right\} \varphi(z) \psi(t) d z d t \\
=\int & \left\{G \partial_{1}(\theta a)\left[\left(-g_{12} G \partial_{2}+g_{22} G \partial_{1}\right)(\theta a)\right](t, z)\right. \\
+ & \left.+G \partial_{2}(\theta a)\left[\left(-g_{11} G \partial_{2}+g_{12} G \partial_{1}\right)(\theta a)\right](t, z)\right\} \varphi(z) \psi(t) d z d t
\end{aligned}
$$

On notera

$$
G(z, Z)=\mathscr{F}_{\zeta}^{-1}\left(-(\operatorname{det} g(z))^{-1}\left(g^{i j}(z) \zeta_{i} \zeta_{j}\right)^{-1} \gamma(\zeta)\right)
$$

le noyau de l'opérateur pseudo-différentiel $G$ de degré -2 sur $\mathbb{R}^{2}$ de symbole (2.2.4). En particulier, pour tout compact $K$ de $\mathbb{R}^{2} \times \mathbb{R}^{2}$, il existe $C>0$ telle 
que

$$
\begin{gathered}
|G(z, Z)| \leq C(1+|\log | Z||), \\
\left|\partial_{Z} G(z, Z)\right| \leq C /|Z|, \quad \forall(z, Z) \in K .
\end{gathered}
$$

Pour prouver les relations $(2.2 .8)_{k},(2.2 .9)$ remarquons que l'on a pour tout $t \in \mathbb{R}$

$$
\begin{aligned}
& \int G \partial_{k}\left(\theta a^{\varepsilon}\right)\left[\left(-g_{1 k} G \partial_{2}+g_{2 k} G \partial_{1}\right)\left(\theta a^{\varepsilon}\right)\right](t, z) \varphi(z) d z \\
& =\int H_{\varphi}^{k}(x, y)\left(\theta a^{\varepsilon}\right)(t, x)\left(\theta a^{\varepsilon}\right)(t, y) d x d y, \quad k=1,2, \\
& \int\left\{G \partial_{1}\left(\theta a^{\varepsilon}\right)\left[\left(-g_{12} G \partial_{2}+g_{22} G \partial_{1}\right)\left(\theta a^{\varepsilon}\right)\right](t, z)\right. \\
& \left.\quad+G \partial_{2}\left(\theta a^{\varepsilon}\right)\left[\left(-g_{11} G \partial_{2}+g_{12} G \partial_{1}\right)\left(\theta a^{\varepsilon}\right)\right](t, z)\right\} \varphi(z) d z \\
& =\int H_{\varphi}^{3}(x, y)\left(\theta a^{\varepsilon}\right)(t, x)\left(\theta a^{\varepsilon}\right)(t, y) d x d y,
\end{aligned}
$$

où $H_{\varphi}^{k}$ est la distribution

$$
\begin{aligned}
H_{\varphi}^{k}(x, y)= & -\frac{\partial^{2}}{\partial x^{k} \partial y^{2}} \int G(z, z-x) G(z, z-y) g_{1 k}(z) \varphi(z) d z \\
& +\frac{\partial^{2}}{\partial x^{k} \partial y^{1}} \int G(z, z-x) G(z, z-y) g_{2 k}(z) \varphi(z) d z
\end{aligned}
$$

pour $k=1,2$ et

$$
\begin{aligned}
H_{\varphi}^{3}(x, y)= & \left(\frac{\partial^{2}}{\partial x^{2} \partial y^{1}}-\frac{\partial^{2}}{\partial x^{1} \partial y^{2}}\right) \int G(z, z-x) G(z, z-y) g_{12}(z) \varphi(z) d z \\
& +\frac{\partial^{2}}{\partial x^{1} \partial y^{1}} \int G(z, z-x) G(z, z-y) g_{22}(z) \varphi(z) d z \\
& -\frac{\partial^{2}}{\partial x^{2} \partial y^{2}} \int G(z, z-x) G(z, z-y) g_{11}(z) \varphi(z) d z
\end{aligned}
$$

La preuve de la Proposition 2.2.3 repose sur le lemme suivant:

Lemme 2.2.4. Les distributions $H_{\varphi}^{k}, k=1,2,3$, sont des fonctions bornées, continues hors de la diagonale, au voisinage de $\operatorname{Supp} \theta \times \operatorname{Supp} \theta$ dans $\mathbb{R}^{2} \times \mathbb{R}^{2}$.

La continuité des fonctions étudiées hors de la diagonale est immédiate compte tenu de la seconde inégalité (2.2.11). Pour prouver que ces fonctions sont bornées, nous utiliserons le résultat suivant:

Lemme 2.2.5. Soient $b_{1}(z, \zeta), b_{2}(z, \zeta)$ deux symboles d'opérateurs pseudodifférentiels d'ordre -1 sur $\mathbb{R}^{2}$ et $\varphi \in C_{0}^{\infty}\left(\mathbb{R}^{2}\right)$. Alors la transformée de Fourier inverse en $(\xi, \eta)$ de la fonction

$$
\int e^{-i z \cdot(\xi+\eta)} b_{1}(z, \xi)\left(b_{2}(z, \eta)-b_{2}(z,-\xi)\right) \varphi(z) d z
$$

est une fonction continue. 
Démonstration. On a

$$
\begin{aligned}
b_{2}(z, \eta)-b_{2}(z,-\xi) & =(\xi+\eta) \cdot \int_{0}^{1} \frac{\partial b_{2}}{\partial \zeta}(z, t \eta-(1-t) \xi) d t \\
& =(\xi+\eta) \cdot \tilde{b}_{2}(z ; \xi, \eta)
\end{aligned}
$$

et pour tout $\alpha \in \mathbb{N}^{2},\left|\partial_{z}^{\alpha} \tilde{b}_{2}(z ; \xi, \eta)\right| \leq C_{\alpha}(1+|\xi|)^{-2}(1+|\xi+\eta|)^{2}$. Alors pour tout entier $N,(2.2 .16)$ se majore en module par

$$
\begin{aligned}
& C_{N}(1+|\xi+\eta|)^{-N} \sum_{|\alpha| \leq N}\left|\int e^{-i z \cdot(\xi+\eta)} \partial_{z}^{\alpha}\left(b_{1}(z, \xi)(\xi+\eta) \cdot \tilde{b}_{2}(z ; \xi, \eta) \varphi(z)\right) d z\right| \\
& \quad \leq C_{N}(1+|\xi+\eta|)^{-N+3}(1+|\xi|)^{-3} .
\end{aligned}
$$

Le résultat en découle si $N$ est pris assez grand.

Démonstration du Lemme 2.2.4. La transformée de Fourier de $H_{\varphi}^{k}$ est égale à

$$
\int e^{-i z(\xi+\eta)} \sigma(G)(z, \xi) \xi_{k} \sigma(G)(z, \eta)\left(g_{1 k}(z) \eta_{2}-g_{2 k}(z) \eta_{1}\right) \varphi(z) d z
$$

si $k=1$ ou 2 et à

$$
\begin{aligned}
& \int e^{-i z(\xi+\eta)} \sigma(G)(z, \xi) \sigma(G)(z, \eta) \\
& \quad \times\left[\xi_{1}\left(g_{12} \eta_{2}-g_{22} \eta_{1}\right)+\xi_{2}\left(g_{11} \eta_{2}-g_{12} \eta_{1}\right)\right] \varphi(z) d z
\end{aligned}
$$

si $k=3$.

Compte tenu du Lemme 2.2.5, il nous faut montrer que la transformée de Fourier inverse en $\xi$ (calculée au point $x-y$ ) des fonctions

$$
\begin{gathered}
\sigma(G)(y, \xi)^{2} \xi_{k}\left(g_{1 k}(y) \xi_{2}-g_{2 k}(y) \xi_{1}\right) \varphi(y), \quad k=1,2, \\
\sigma(G)(y, \xi)^{2}\left[\xi_{1}\left(g_{12}(y) \xi_{2}-g_{22}(y) \xi_{1}\right)+\xi_{2}\left(g_{11}(y) \xi_{2}-g_{12}(y) \xi_{1}\right)\right] \varphi(y)
\end{gathered}
$$

est bornée en $(x, y)$. Or d'après l'expression (2.2.4) de $\sigma(G)$ et (2.1.29) ces quantités valent respectivement

$$
\begin{aligned}
& \gamma(\xi)^{2}(\operatorname{det} g(y))^{-1}\left(\sum_{i, j} g^{i j} \xi_{i} \xi_{j}\right)^{-2}\left(g^{22}(y) \xi_{2}+g^{12}(y) \xi_{1}\right) \xi_{1} \varphi(y), \\
& -\gamma(\xi)^{2}(\operatorname{det} g(y))^{-1}\left(\sum_{i, j} g^{i j} \xi_{i} \xi_{j}\right)^{-2}\left(g^{12}(y) \xi_{2}+g^{11}(y) \xi_{1}\right) \xi_{2} \varphi(y), \\
& \gamma(\xi)^{2}(\operatorname{det} g(y))^{-1}\left(\sum_{i, j} g^{i j} \xi_{i} \xi_{j}\right)^{-2} \varphi(y) \\
& \quad \times\left[-\xi_{1}\left(g^{12}(y) \xi_{2}+g^{11}(y) \xi_{1}\right)+\xi_{2}\left(g^{22}(y) \xi_{2}+g^{12}(y) \xi_{1}\right)\right]
\end{aligned}
$$

donc sont égales au produit de $\gamma(\xi)^{2}(\operatorname{det} g(y))^{-1} \varphi(y)$ par les distributions 
respectives

$$
\begin{gathered}
-\frac{1}{2} \frac{\partial}{\partial \xi_{2}}\left[\xi_{1}\left(\sum_{i, j} g^{i j} \xi_{i} \xi_{j}\right)^{-1}\right], \\
\frac{1}{2} \frac{\partial}{\partial \xi_{1}}\left[\xi_{2}\left(\sum_{i, j} g^{i j} \xi_{i} \xi_{j}\right)^{-1}\right], \\
\frac{1}{2} \frac{\partial}{\partial \xi_{1}}\left[\xi_{1}\left(\sum_{i, j} g^{i j} \xi_{i} \xi_{j}\right)^{-1}\right]-\frac{1}{2} \frac{\partial}{\partial \xi_{2}}\left[\xi_{2}\left(\sum_{i, j} g^{i j} \xi_{i} \xi_{j}\right)^{-1}\right] .
\end{gathered}
$$

En dimensoin 2, la transformée de Fourier inverse d'une fonction homogène de degré -1 est homogène de degré -1 . La transformée de Fourier inverse de chacune des expressions précédentes s'exprime donc à partir du produit de $x^{1}$ ou $x^{2}$ par une fonction homogène de degré -1 : c'est donc une fonction bornée. Le Lemme 2.2.4 s'en déduit immédiatement.

Fin de la preuve de la Proposition 2.2.3. D'après (2.2.12), (2.2.13), on doit étudier l'expression

$$
\int H_{\varphi}^{k}(x, y)\left(\theta a^{\varepsilon}\right)(t, x)\left(\theta a^{\varepsilon}\right)(t, y) d x d y \psi(t) d t
$$

Les hypothèses du Théorème 2.2.1 assurent que $\theta a^{\varepsilon}$ satisfait aux hypothèses de la Proposition 1.2.6. Comme, d'après le Lemme 2.2.4, les fonctions $H_{\varphi}^{k}$ sont continues hors de la diagonale et bornées, la Proposition 1.2.6 entraîne (cf. la remarque suivant sa preuve):

$$
\begin{array}{r}
\lim _{\substack{\varepsilon \rightarrow 0 \\
\varepsilon \in J}} \int H_{\varphi}^{k}(x, y)\left(\theta a^{\varepsilon}\right)(t, x)\left(\theta a^{\varepsilon}\right)(t, y) \psi(t) d x d y d t \\
=\int H_{\varphi}^{k}(x, y) \psi(t) \theta(x) \theta(y) d a_{t}(x) \otimes d a_{t}(y) d t
\end{array}
$$

où $a_{t}=a(t, \cdot)$ est la mesure limite de $a^{\varepsilon}(t, \cdot)$ et où le membre de droite a un sens grâce au Lemme 2.2.4 et au fait que la diagonale $x=y$ est de mesure nulle pour $d\left|a_{t}\right|(x) \otimes d\left|a_{t}\right|(y)$ (cf. la justification de l'expression (1.2.21)). Mais d'autre part, le membre de droite de (2.2.26) n'est autre que le membre de droite de $(2.2 .8)_{k}-(2.2 .9)$ comme on le voit en appliquant le raisonnement précédent à la suite $\theta \underline{a}^{\varepsilon}(t, x)$, obtenue en régularisant $a(t, \cdot)$ par rapport à $x$, qui est bornée dans $L^{\infty}\left(\mathbb{R}, H^{-1}\left(\mathbb{R}^{2}\right)\right)$ et qui, pour presque tout $t$, converge vers $\theta a_{t}$ fortement dans $H^{-1}\left(\mathbb{R}^{2}\right.$ ) (cf. fin du paragraphe 1.2). La Proposition 2.2.3 est donc prouvée et par voie de conséquence le Théorème 2.2.1 également.

2.3. Nappes de tourbillon sur un ouvert à bord. Dans ce dernier paragraphe, nous nous proposons de montrer que les résultats précédents admettent un analogue pour l'équation d'Euler sur un ouvert à bord de $\mathbb{R}^{2}$. Soit $\Omega$ un ouvert borné de $\mathbb{R}^{2}$ à bord $C^{\infty}$, localement d'un seul côté de son bord. On notera $n$ la normale unitaire intérieure à $\partial \Omega$. Pour tout $s$ réel, on désignera par $H^{S}(\Omega)$, 
(resp. $\left.H^{s}\left(\Omega ; \mathbb{R}^{2}\right)\right)$ l'espace des restrictions à $\Omega$ d'applications de classe $H^{s}$ définies au voisinage de $\bar{\Omega}$ à valeurs réelles (resp. à valeurs dans $\mathbb{R}^{2}$ ). De même $C^{\infty}(\bar{\Omega})$ (resp. $C^{\infty}\left(\bar{\Omega} ; \mathbb{R}^{2}\right)$ ) est l'espace des applications $C^{\infty}$ jusqu'au bord dans $\Omega$ à valeurs dans $\mathbb{R}$ (resp. dans $\mathbb{R}^{2}$ ). Si $v$ est un élément de $H^{s}\left(\Omega ; \mathbb{R}^{2}\right)$ avec $\left.s \in\right]-\frac{1}{2}, \frac{1}{2}\left[\right.$, on notera $v^{\#}$ son prolongement par 0 hors de $\Omega$ : c'est un élément de $H^{s}\left(\mathbb{R}^{2} ; \mathbb{R}^{2}\right)$. Nous allons prouver

Théorème 2.3.1. Soit $v_{0} \in L^{2}\left(\Omega ; \mathbb{R}^{2}\right)$ vérifiant $\operatorname{div} v_{0}=0,\left.\left(v_{0} \cdot n\right)\right|_{\partial \Omega}=0$. Supposons que le tourbillon $\omega_{0}=\operatorname{rot} v_{0}$ de $v_{0}$ s'écrive $\omega_{0}=\omega_{0}^{\prime}+\omega_{0}^{\prime \prime}$ où $\omega_{0}^{\prime}$ est la restriction à $\Omega$ d'une mesure positive ou nulle sur $\mathbb{R}^{2}$, appartenant à $H^{-1}\left(\mathbb{R}^{2}\right)$, à support compact et où $\omega_{0}^{\prime \prime} \in L^{p}(\Omega)$ pour un réel $p>1$. Il existe alors $v \in L^{\infty}\left(\mathbb{R}, L^{2}\left(\Omega ; \mathbb{R}^{2}\right)\right)$ et $p \in L^{\infty}\left(\mathbb{R}, \mathscr{D}^{\prime}(\Omega)\right)$ solutions du système:

$$
\begin{cases}\partial v / \partial t+\operatorname{div}(v \otimes v)=-\nabla p & \text { sur } \mathbb{R} \times \Omega, \\ \operatorname{div} v=0 & \text { sur } \mathbb{R} \times \Omega, \\ \left.(v \cdot n)\right|_{\partial \Omega}=0 & \text { sur } \mathbb{R} \times \partial \Omega, \\ \left.v\right|_{t=0}=v_{0} & \text { sur } \Omega .\end{cases}
$$

En outre, $\omega$ se décompose en $\omega=\omega^{\prime}+\omega^{\prime \prime}$ où $\omega^{\prime}$ (resp. $\left.\omega^{\prime \prime}\right)$ est fonction $L^{\infty}$ de $t$ à valeurs dans les mesures positives ou nulles sur $\Omega$ (resp. à valeurs dans $L^{p}(\Omega)$ et vérifie $\left.\left\|\omega^{\prime \prime}\right\|_{L^{\infty}\left(\mathbb{R}, L^{p}(\Omega)\right)} \leq\left\|\omega_{0}^{\prime \prime}\right\|_{L^{p}(\Omega)}\right)$.

Il est bien connu que la condition aux limites $\left.(v \cdot n)\right|_{\partial \Omega}=0$ a un sens pour un élément $v$ de $L^{2}\left(\Omega ; \mathbb{R}^{2}\right)$ vérifiant $\operatorname{div} v=0$. On a en effet

Lemme 2.3.2. Soit $s>-\frac{1}{2}$. L'application $\left.v \rightarrow(v \cdot n)\right|_{\partial \Omega}$ définie sur $C^{\infty}\left(\bar{\Omega} ; \mathbb{R}^{2}\right)$ se prolonge en une application continue de

$$
\left\{v \in H^{s}\left(\Omega ; \mathbb{R}^{2}\right) ; \operatorname{div} v=0\right\}
$$

$\grave{a}$ valeurs dans $H^{s-1 / 2}(\partial \Omega)$.

Démonstration. Le cas $s>\frac{1}{2}$ est trivial. On peut donc supposer $\left.s \in\right]-\frac{1}{2}, \frac{1}{2}[$ (le cas $s=\frac{1}{2}$ s'obtenant ensuite par interpolation). Alors, si $v \in C^{\infty}\left(\bar{\Omega} ; \mathbb{R}^{2}\right)$ vérifie $\operatorname{div} v=0$ on a

$$
\begin{aligned}
\left\|\left.(v \cdot n)\right|_{\partial \Omega}\right\|_{H^{s-1 / 2}(\partial \Omega)} & \leq C\left\|\left.(v \cdot n)\right|_{\partial \Omega} \otimes \delta_{\partial \Omega}\right\|_{H^{s-1}\left(\mathbb{R}^{2}\right)} \\
& \leq C\left\|\operatorname{div}\left(v^{\#}\right)\right\|_{H^{s-1}\left(\mathbb{R}^{2}\right)} \\
& \leq C\left\|v^{\#}\right\|_{H^{s}\left(\mathbb{R}^{2} ; \mathbb{R}^{2}\right)} \leq C\|v\|_{H^{s}\left(\Omega ; \mathbb{R}^{2}\right)},
\end{aligned}
$$

d'où le résultat.

D'après [7, Théorème 4.3.3] et la décomposition de Hodge-Kodaïra [7, p. 191], l'application

$$
\begin{aligned}
\left\{v \in L^{2}\left(\Omega ; \mathbb{R}^{2}\right) ; \operatorname{div} v=0,\left.(v \cdot n)\right|_{\partial \Omega}=0\right\} & \rightarrow H^{-1}(\Omega), \\
v & \rightarrow \operatorname{rot} v
\end{aligned}
$$


est surjective. En outre [7, Théorèmes 4.2.2 et 3.2.3] son noyau est l'espace de dimension finie des champs harmoniques tangents à $\partial \Omega$, i.e.,

$$
\left\{h \in C^{\infty}\left(\bar{\Omega} ; \mathbb{R}^{2}\right) ; \operatorname{div} h=0, \operatorname{rot} h=0,\left.(h \cdot n)\right|_{\partial \Omega}=0\right\} .
$$

Si $E$ désigne l'orthogonal du noyau de (2.3.3) et $L: H^{-1}(\Omega) \rightarrow E$ l'inverse de la restriction de (2.3.3) à $E$, les données du problème (2.3.1) vérifient $v_{0}=$ $L\left(\omega_{0}\right)+h_{0}$ où $h_{0}$ est un champ harmonique tangent à $\partial \Omega$ convenable.

Soit $\rho \in C_{0}^{\infty}\left(\mathbb{R}^{2}\right), \rho \geq 0, \int \rho(x) d x=1$ et pour $\left.\varepsilon \in\right] 0,1\left[, \rho_{\varepsilon}(x)=\right.$ $\varepsilon^{-2} \rho(x / \varepsilon)$. Soient $\omega_{0}^{\text {b }}$ une mesure positive élément de $H_{\text {comp }}^{-1}\left(\mathbb{R}^{2}\right)$ telle que $\left.\omega_{0}^{\prime b}\right|_{\Omega}=\omega_{0}^{\prime}$ et $\omega_{0}^{\prime \prime b}$ le prolongement de $\omega_{0}^{\prime \prime}$ par 0 hors de $\Omega$. On pose alors

$$
\omega_{0}^{\prime \varepsilon}=\left.\left(\omega_{0}^{\prime b} * \rho_{\varepsilon}\right)\right|_{\Omega}, \quad \omega_{0}^{\prime \prime \varepsilon}=\left.\left(\omega_{0}^{\prime \prime b} * \rho_{\varepsilon}\right)\right|_{\Omega}, \quad \omega_{0}^{\varepsilon}=\omega_{0}^{\prime \varepsilon}+\omega_{0}^{\prime \prime \varepsilon} .
$$

Alors $\left(\omega_{0}^{\prime \varepsilon}\right)_{\varepsilon \in] 0,1]}$ est une suite de fonctions $C^{\infty}$ sur $\bar{\Omega}$ positives ou nulles, bornée dans $L^{1}(\Omega) \cap H^{-1}(\Omega)$ et convergeant vers $\omega_{0}^{\prime}$ dans $H^{-1}(\Omega)$ lorsque $\varepsilon \rightarrow 0$. De même $\left(\omega_{0}^{\prime \prime \varepsilon}\right)_{\varepsilon \in] 0,1]}$ est bornée dans $L^{p}(\Omega)$ par $\left\|\omega_{0}^{\prime \prime}\right\|_{L^{p}(\Omega)}$ et converge dans cet espace vers $\omega_{0}^{\prime \prime}$ lorsque $\varepsilon \rightarrow 0$. On pose

$$
v_{0}^{\varepsilon}=L\left(\omega_{0}^{\varepsilon}\right)+h_{0} .
$$

C'est une suite de fonction $C^{\infty}$ sur $\bar{\Omega}$, bornée dans $L^{2}\left(\Omega ; \mathbb{R}^{2}\right)$ convergeant dans cet espace vers $v_{0}$ lorsque $\varepsilon$ tend vers 0 , vérifiant $\operatorname{div} v_{0}^{\varepsilon}=0,\left.\left(v_{0}^{\varepsilon} \cdot n\right)\right|_{\partial \Omega}=$ 0 . D'après $[9 ; 6, \S 15]$, le problème

$$
\left\{\begin{array}{l}
\partial v^{\varepsilon} / \partial t+\left(v^{\varepsilon} \cdot \nabla\right) v^{\varepsilon}=-\nabla p^{\varepsilon} \\
\operatorname{div} v^{\varepsilon}=0 \\
\left.\left(v^{\varepsilon} \cdot n\right)\right|_{\partial \Omega}=0 \\
\left.v^{\varepsilon}\right|_{t=0}=v_{0}^{\varepsilon}
\end{array}\right.
$$

admet une unique solution globale $C^{\infty}$ sur $\mathbb{R} \times \bar{\Omega}, v^{\varepsilon}(t, x)$. En outre, on a l'inégalité d'énergie

$$
\left\|v^{\varepsilon}\right\|_{L^{\infty}\left(\mathbb{R}, L^{2}\left(\Omega ; \mathbb{R}^{2}\right)\right)} \leq\left\|v_{0}^{\varepsilon}\right\|_{L^{2}\left(\Omega ; \mathbb{R}^{2}\right)}
$$

et donc $\left(v^{\varepsilon}\right)_{\varepsilon}$ est uniformément bornée dans $L^{\infty}\left(\mathbb{R}, L^{2}\left(\Omega ; \mathbb{R}^{2}\right)\right)$. Notons $\omega^{\varepsilon}=$ $\operatorname{rot} v^{\varepsilon}$. On a alors $\omega^{\varepsilon}=\omega^{\ell}+\omega^{\prime \ell}$, où $\omega^{\ell}$ et $\omega^{\prime \prime}$ sont respectivement définis par:

$$
\begin{aligned}
& \left\{\begin{array}{l}
\partial \omega^{\prime \ell} / \partial t+\left(v^{\varepsilon} \cdot \nabla\right) \omega^{\prime \varepsilon}=0, \\
\left.\omega^{\prime \varepsilon}\right|_{t=0}=\omega_{0}^{\prime \varepsilon} ;
\end{array}\right. \\
& \left\{\begin{array}{l}
\partial \omega^{\prime \ell} / \partial t+\left(v^{\varepsilon} \cdot \nabla\right) \omega^{\prime \ell}=0, \\
\left.\omega^{\prime \prime}\right|_{t=0}=\omega_{0}^{\prime \ell} ;
\end{array}\right.
\end{aligned}
$$

et comme $v^{\varepsilon}$ est un champ tangent à $\partial \Omega$ et à divergence nulle, on a

$$
\begin{aligned}
& \omega^{\prime \varepsilon} \geq 0, \quad\left\|\omega^{\prime \varepsilon}\right\|_{L^{\infty}\left(\mathbb{R}, L^{1}(\Omega)\right)} \leq\left\|\omega_{0}^{\prime \varepsilon}\right\|_{L^{1}(\Omega)}, \\
& \left\|\omega^{\prime \prime \varepsilon}\right\|_{L^{\infty}\left(\mathbb{R}, L^{p}(\Omega)\right)} \leq\left\|\omega_{0}^{\prime \prime \varepsilon}\right\|_{L^{p}(\Omega)} \leq\left\|\omega_{0}^{\prime \prime}\right\|_{L^{p}(\Omega)} .
\end{aligned}
$$


Enfin, on remarquera que $\left(v^{\varepsilon}\right)_{\varepsilon \in] 0,1]}$ est bornée dans $\operatorname{Lip}\left(\mathbb{R}, \mathscr{D}^{\prime}\left(\Omega ; \mathbb{R}^{2}\right)\right)$. En effet, si $\Phi \in C_{0}^{\infty}\left(\Omega ; \mathbb{R}^{2}\right)$ et si l'on écrit la décomposition de Hodge-Kodaïra de $\Phi$ :

$$
\Phi=\Psi+\nabla f
$$

avec $\Psi \in C^{\infty}\left(\bar{\Omega} ; \mathbb{R}^{2}\right), \operatorname{div} \Psi=0,\left.(\Psi \cdot n)\right|_{\partial \Omega}=0$ et $f \in C^{\infty}(\bar{\Omega})$, on a

$$
\begin{aligned}
\frac{\partial}{\partial t}\left\langle v^{\varepsilon}(t, \cdot), \Phi\right\rangle & =\frac{\partial}{\partial t}\left\langle v^{\varepsilon}(t, \cdot), \Psi\right\rangle \\
& =-\left\langle\operatorname{div}\left(v^{\varepsilon} \otimes v^{\varepsilon}(t, \cdot)\right), \Psi\right\rangle
\end{aligned}
$$

quantité qui est uniformément bornée en $t$ et $\varepsilon$ lorsque $\Phi$ décrit une partie bornée de $C_{0}^{\infty}\left(\Omega ; \mathbb{R}^{2}\right)$. Grâce aux inégalités (2.3.6), (2.3.9), il existe $\left.J \subset\right] 0,1[$ avec $0 \in \bar{J}$ tel que les suites $\left(v^{\varepsilon}\right)_{\varepsilon \in J},\left(\omega^{\varepsilon}\right)_{\varepsilon \in J},\left(\omega^{\prime \varepsilon}\right)_{\varepsilon \in J},\left(\omega^{\prime \prime}\right)_{\varepsilon \in J}$ convergent au sens des distributions sur $\mathbb{R} \times \Omega$ vers des limites $v, \omega, \omega^{\prime}, \omega^{\prime \prime}$. On a $v \in$ $L^{\infty}\left(\mathbb{R}, L^{2}\left(\Omega ; \mathbb{R}^{2}\right)\right), \omega=\omega^{\prime}+\omega^{\prime \prime}, \omega^{\prime}$ est fonction $L^{\infty}$ de $t$ à valeurs dans les mesures positives ou nulles sur $\Omega$ qui sont dans $H^{-1}(\Omega), \omega^{\prime \prime} \in L^{\infty}\left(\mathbb{R}, L^{p}(\Omega)\right)$ avec une norme dans cet espace majorée par $\left\|\omega_{0}^{\prime \prime}\right\|_{L^{p}(\Omega)}$.

Si $\varphi \in C_{0}^{\infty}(\Omega), \psi \in C_{0}^{\infty}(\mathbb{R})$, remarquons alors que le Théorème 2.2.1 s'applique aux intégrales

$$
\begin{gathered}
\int v_{1}^{\varepsilon}(t, z) v_{2}^{\varepsilon}(t, z) \varphi(z) \psi(t) d z d t \\
\int\left[v_{1}^{\varepsilon}(t, z)^{2}-v_{2}^{\varepsilon}(t, z)^{2}\right] \varphi(z) \psi(t) d z d t
\end{gathered}
$$

(le fait que l'on soit ici sur une variété à bord $\Omega$ ne joue pas puisqu'on ne s'intéresse qu'à un résultat local à l'intérieur). Il en résulte donc que $v_{1}^{\varepsilon} \cdot v_{2}^{\varepsilon}$ et $\left(v_{1}^{\varepsilon}\right)^{2}-\left(v_{2}^{\varepsilon}\right)^{2}$ convergent au sens des distributions sur $\mathbb{R} \times \Omega$ vers $v_{1} \cdot v_{2}$ et $v_{1}^{2}-v_{2}^{2}$ respectivement.

Utilisons alors que si $\left(v^{\varepsilon}, p^{\varepsilon}\right)$ est solution de $(2.3 .5),\left(v^{\varepsilon}, q^{\varepsilon}=p^{\varepsilon}+\frac{1}{2}\left|v^{\varepsilon}\right|^{2}\right)$ vérifie

$$
\left\{\begin{array}{l}
\frac{\partial v^{\varepsilon}}{\partial t}+B(D)\left(\begin{array}{c}
\left(v_{1}^{\varepsilon}\right)^{2}-\left(v_{2}^{\varepsilon}\right)^{2} \\
v_{1}^{\varepsilon} \cdot v_{2}^{\varepsilon}
\end{array}\right)=-\nabla q^{\varepsilon} \\
\operatorname{div} v^{\varepsilon}=0 \\
\left.\left(v^{\varepsilon} \cdot n\right)\right|_{\partial \Omega}=0 \\
\left.v^{\varepsilon}\right|_{t=0}=v_{0}^{\varepsilon}
\end{array}\right.
$$

avec

$$
B(D)=\left(\begin{array}{cc}
\frac{1}{2} \partial_{1} & \partial_{2} \\
-\frac{1}{2} \partial_{2} & \partial_{1}
\end{array}\right) .
$$

Le membre de gauche de (2.3.13) converge d'après ce que l'on vient de voir vers

$$
\frac{\partial v}{\partial t}+B(D)\left(\begin{array}{c}
v_{1}^{2}-v_{2}^{2} \\
v_{1} v_{2}
\end{array}\right)
$$


Or, d'après un théorème de de Rham [11, Théorème $\left.17^{\prime}, \S 22\right]$, l'espace des formes différentielles exactes à coefficients distributions sur un ouvert est fermé pour la topologie faible. Il existe donc pour tout $t \in \mathbb{R}$, une distribution $q(t, \cdot)$ sur $\Omega$ telle que (2.3.14) soit égal à $-\nabla q$. Bien entendu, puisque (2.3.14) est borné à valeurs distributions, on peut supposer que $q \in L^{\infty}\left(\mathbb{R}, \mathscr{D}^{\prime}(\Omega)\right)$. Si l'on pose $p=q-\frac{1}{2} v^{2}$ on voit donc que la première équation (2.3.1) est satisfaite par $(v, p)$. D'autre part, puisque pour tout $t, v^{\varepsilon}(t, \cdot)$ converge vers $v(t, \cdot)$ fortement dans $H^{s}(\Omega)$ pour tout $s<0$, il résulte du Lemme 2.3.2 que $\left.\left(v^{\varepsilon} \cdot n\right)\right|_{\partial \Omega}$ converge au sens des distributions sur $\mathbb{R} \times \partial \Omega$ vers $\left.(v \cdot n)\right|_{\partial \Omega}$. La troisième équation (2.3.1) est donc satisfaite. Le Théorème 2.3.1 est donc démontré.

\section{BIBLIOGRAPHIE}

1. R. Abraham, J. E. Marsden, et T. Ratiu, Manifolds, tensor analysis and applications, Appl. Math. Sci., vol. 75, 2nd ed., Springer-Verlag, 1988.

2. S. Alinhac, Un phénomène de concentration évanescente pour des flots nonstationnaires incompressibles en dimension deux, Prépublications de l'Université Paris-Sud.

3. J.-Y. Chemin, Sur le mouvement des particules d'un fluide parfait, incompressible, bidimensionnel, Invent. Math. 103 (1991), 599-629.

4. R. Di Perna et A. Majda, Concentrations in regularizations for 2-D incompressible flow, Comm. Pure Appl. Math. 40 (1987), 301-345.

5. __ Reduced Hausdorff dimension and concentration-cancellation for two dimensional incompressible flow, J. Amer. Math. Soc. 1 (1988), 59-95.

6. D. Ebin et J. Marsden, Groups of diffeomorphisms and the motion of an incompressible fluid, Ann. of Math. (2) 92 (1970), 102-163.

7. V. Georgescu, Some boundary value problems for differential forms on compact Riemannian manifolds, Ann. Mat. Pura Appl. (4) 122 (1979), 159-198.

8. C. Greengard et E. Thomann, On Di Perna-Majda concentration sets for two-dimensional incompressible flow, Comm. Pure Appl. Math. 41 (1988), 295-303.

9. T. Kato, On classical solutions of the two dimensional non-stationary Euler equation, Arch. Rational Mech. Anal. 25 (1967), 188-200.

10. F. Mac Grath, Non-stationary plane flow of viscous and ideal fluids, Arch. Rational Mech. Anal. 27 (1968), 328-348.

11. G. de Rham, Variétés différentiables, Hermann, Actualités Sci. Indust., Paris, 1960.

12. Yuxi Zheng, Concentration-cancellation for the velocity fields in two dimensional incompressible fluid flows, preprint, Univ. of California at Berkeley.

13. R. Krasny, Desingularization of periodic vortex sheet roll-up, J. Comput. Phys. 65 (1986), 292-313.

14. _ Computation of vortex sheet roll-up in the Treftz plane, J. Fluid Mech. 184 (1987), 123-155.

15. A. Majda, Vortex dynamics: Numerical analysis, scientific computing, and mathematical theory, Proceedings of the First International Congress for Industrial and Applied Mathematics, S.I.A.M. Publ., 1988, pp. 153-182.

16. _ - The interaction of nonlinear analysis and modern applied mathematics, Proc. I.C.M., Kyoto (à paraître).

Département de Mathematiques, Université de Paris-Sud, 91405 Orsay Cedex, France

Adresse á partir du $1^{\text {er }}$ Septembre 1991: C. S. P., Département de Mathématiques et Informatique, Université Paris XIII, Avenue Jean-Baptiste Elément, 93430 Villetaneuse, France 